\title{
Orphan CpG islands boost the regulatory activity of poised enhancers and dictate the responsiveness of their target genes.
}

\section{Authors}

Tomás Pachano ${ }^{1}$, Víctor Sánchez-Gaya², María Mariner-Faulî2, Thais Ealo², Helena G. Asenjo $^{3,4,5}$, Patricia Respuela ${ }^{2}$, Sara Cruz-Molina ${ }^{6}$, Wilfred F. J. van Ijcken ${ }^{7}$, David Landeira $^{3,4,5}$ and Álvaro Rada-Iglesias ${ }^{1,2,8^{*}}$.

\section{Affiliations}

${ }^{1}$ Center for Molecular Medicine Cologne (CMMC), University of Cologne, Robert-KochStrasse 21, 50931 Cologne, Germany

${ }^{2}$ Institute of Biomedicine and Biotechnology of Cantabria (IBBTEC), CSIC/Universidad de Cantabria, Albert Einstein 22, 39011 Santander, Spain

${ }^{3}$ Centre for Genomics and Oncological Research (GENYO), Avenue de la Ilustración 114, 18016 Granada, Spain.

${ }^{4}$ Department of Biochemistry and Molecular Biology II, Faculty of Pharmacy, University of Granada, Granada, Spain

${ }^{5}$ Instituto de Investigación Biosanitaria ibs.GRANADA, Hospital Virgen de las Nieves, Granada, Spain

${ }^{6}$ Max Planck Institute for Molecular Biomedicine, Roentgenstrasse 20, 48149 Muenster, Germany.

${ }^{7}$ Erasmus Medical Center, University of Rotterdam, Rotterdam, the Netherlands.

${ }^{8}$ Cologne Excellence Cluster for Cellular Stress Responses in Aging-Associated Diseases (CECAD), University of Cologne, Joseph-Stelzmann-Strasse 26, 50931 Cologne, Germany

* corresponding author: alvaro.rada@unican.es 


\section{ARTICLE}

CpG islands (CGIs) represent a distinctive and widespread genetic feature of vertebrate genomes, being associated with $\sim 70 \%$ of all annotated gene promoters ${ }^{1}$. CGIs have been proposed to control transcription initiation by conferring nearby promoters with unique chromatin properties $^{2-4}$. In addition, there are thousands of distal or orphan CGIs (oCGIs) whose functional relevance and mechanism of action are barely $\mathbf{k n o w n}^{5-7}$. Here we show that oCGIs are an essential component of poised enhancers (PEs) ${ }^{8,9}$ that boost their long-range regulatory activity and dictate the responsiveness of their target genes. Using a CRISPR/Cas9 knock-in strategy in mESC, we introduced PEs with or without oCGIs within topological associating domains (TADs) harbouring genes with different types of promoters. By evaluating the chromatin, topological and regulatory properties of the engineered PEs, we uncover that, rather than increasing their local activation, oCGIs boost the physical and functional communication between PEs and distally located developmental genes. Furthermore, we demonstrate that developmental genes with CpG rich promoters are particularly responsive to PEs and that such responsiveness depends on the presence of oCGIs. Therefore, our work unveils a novel role for CGIs as genetic determinants of the compatibility between genes and enhancers, thus providing major insights into how developmental gene expression programs are deployed under both physiological and pathological conditions ${ }^{10-12}$.

The establishment of cell-type specific gene expression programs during vertebrate development is largely dependent on enhancers ${ }^{13-15}$, a group of distal cis-regulatory elements containing clusters of transcription factor binding sites (TFBS) that can control gene expression in a distance and orientation independent manner. The regulatory properties of enhancers have been mostly investigated using transgenic reporter assays ${ }^{16}$, in which the enhancer activity of selected sequences is evaluated by measuring their capacity to activate transcription of a reporter gene from a minimal promoter. In these assays, the investigated enhancers are placed at relatively small distances from the reporter genes and using a rather limited set of minimal promoters. On the other hand, the regulatory activity of enhancers can be specifically directed towards their target genes by insulators, which can prevent enhancers from ectopically activating non-target genes ${ }^{10}$. In vertebrates, insulators are typically bound by CTCF, which, together with Cohesin, can form long-range chromatin loops that demarcate the boundaries of regulatory domains (i.e. $\operatorname{TADs}^{17}$, insulated neighborhoods ${ }^{18}$ ) and limit 
enhancer activity towards genes located within the same domain ${ }^{17,19}$. Altogether, current models of enhancer function implicitly assume that enhancers and genes can effectively communicate with each other, regardless of distance or sequence composition, as far as they are located within the same regulatory domain ${ }^{10,11}$. However, these models have been challenged by recent studies showing that the loss or structural disruption of regulatory domains does not always lead to changes in gene expression or enhancer-gene communication $^{20-23}$. Therefore, additional factors, besides being in the same regulatory domain, might contribute to the compatibility between genes and enhancers. In this regard, massively parallel reporter assays uncovered that enhancer responsiveness in flies depends on the sequence composition of core promoter elements ${ }^{24,25}$. However, it is currently unknown whether other genetic factors, such as distance or enhancer sequence composition, can also contribute to such responsiveness. Uncovering the rules controlling the responsiveness of genes to enhancers is essential to understand and predict the pathological consequences of human structural variation ${ }^{12}$.

Focusing on a set of highly conserved developmental enhancers, known as PEs, here we have used a synthetic engineering approach to systematically dissect the genetic rules controlling gene-enhancer compatibility. We previously showed that PEs are essential for the induction of major anterior neural genes upon ESC differentiation ${ }^{9}$. Before becoming active in anterior neural progenitors (AntNPC), PEs are already bookmarked in embryonic stem cells (ESC) with unique chromatin and topological features, including binding by polycomb-group protein complexes $(\mathrm{PcG})$ and pre-formed contacts with their target genes ${ }^{8,9}$. Compared to other enhancer types, PEs display a distinctive genetic composition that includes not only clusters of TFBS but also nearby CGIs. CGIs are a prevalent feature of gene promoters in vertebrates, where they are believed to provide a permissive chromatin state that facilitates transcription initiation $^{2-4}$. However, only half of the CGIs found in the mouse and human genomes are associated to promoters (pCGIs) ${ }^{1,5}$; while the other half, typically known as oCGI, remain poorly studied. oCGIs have been proposed to act as alternative gene promoters ${ }^{6,7}$ or as highly active and conserved enhancers with limited tissue specificity ${ }^{5,26,27}$. Nevertheless, these proposed functions are largely based on correlative observations and the mechanisms whereby oCGIs might contribute to gene expression control remain fully unknown. Here we show that oCGIs act as long-range boosters of PE regulatory activity that enable functional communication between PEs and developmental genes. Notably, we also show that such communication is efficiently established with $\mathrm{CpG}$-rich but not with $\mathrm{CpG}$-poor promoters, 
suggesting that distal sequences, namely oCGIs, can also contribute to enhancer responsiveness and gene expression specificity. Therefore, our work provides major insights into the genetic basis of gene-enhancer compatibility and, thus, into how gene expression programs can be specifically and precisely deployed during development as well as pathologically disrupted by structural variants ${ }^{10-12}$.

\section{Genetic properties of PE-associated oCGIS}

We previously reported that PEs are commonly located in proximity to computationally predicted CGIs ${ }^{9}$. However, computational models underestimate the abundance of CGIs, especially those that are distally located from transcription start sites (TSS) ${ }^{28}$. Therefore, we used biochemically identified non-methylated islands (NMI) to more accurately study the association between PEs and oCGIs ${ }^{28}$ and found that $\sim 80 \%$ of PEs are located within $3 \mathrm{~Kb}$ of a NMI (Fig. 1a). Next, we genetically compared the NMI associated to PEs (PE-NMI) with those found in proximity of the TSS of developmental genes (devTSS-NMI) (see Methods). In line with the previous characterization of intergenic $\mathrm{NMI}^{5,28}$, PE-NMI are shorter in length and present lower CpG density than devTSS-NMI (Fig. 1b). Similar results were observed using an independent set of biochemically defined CGI (i.e. CAP-CGI) (Extended Data Fig. $1)^{5}$. Thus, PEs are pervasively found in proximity of CGIs genetically distinct from those associated with gene promoters.

\section{oCGIs are necessary for the regulatory function of PES}

To start evaluating the regulatory role of oCGIs in the context of PEs, we first generated mESC lines with a homozygous deletion of the oCGI associated with a previously characterized PE (i.e. PE Soxl $(+35)^{9}$ ) that controls the expression of Soxl in neural progenitors (PE Soxl $(+35)-C G I^{-/}$mESC; Fig. 1c and Extended Data Fig. 2a). ChIP-qPCR experiments in mESC showed that the deletion of the oCGI severely reduced H3K27me3 levels around the PE Soxl(+35) region (Extended Data Fig. 2b), thus demonstrating that oCGIs are necessary for the recruitment of PcG to PE. Next, to investigate how the loss of the oCGI might affect PE Soxl(+35) function, we measured Soxl expression in WT, $P E$ $\operatorname{Soxl}(+35)-C G I^{-/}$and $P E \operatorname{Soxl}(+35)^{-/} \mathrm{mESC}$ as well as upon their differentiation into AntNPC. In mESC, neither the deletion of the oCGI nor of the whole PE Soxl(+35) affected Soxl expression (Fig. 1d; Extended Data Fig. 2c). Together with our previous work ${ }^{9}$, these results further support that PEs do not act as silencers in ESC and that PcG recruitment is not required to keep PEs is an inactive sate. However, upon differentiation into AntNPC, the 


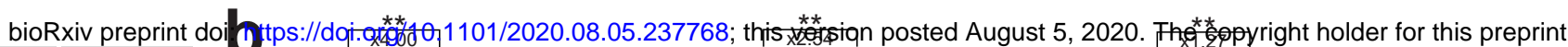

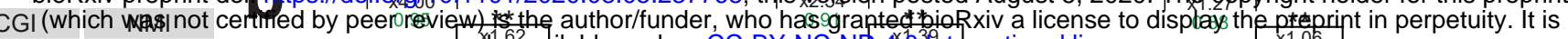

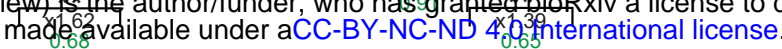

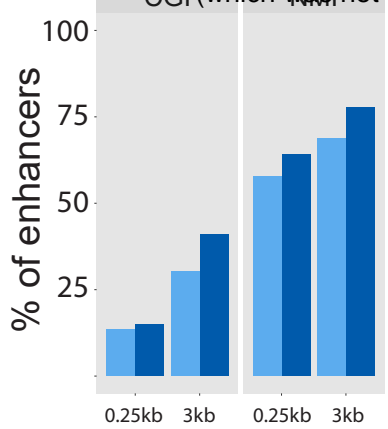

Max. distance

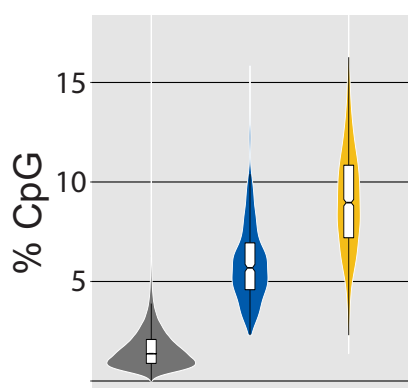

PE-all

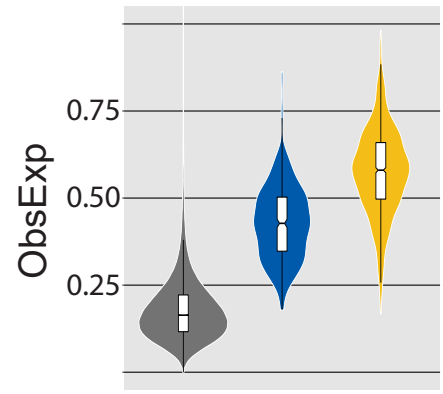

Random

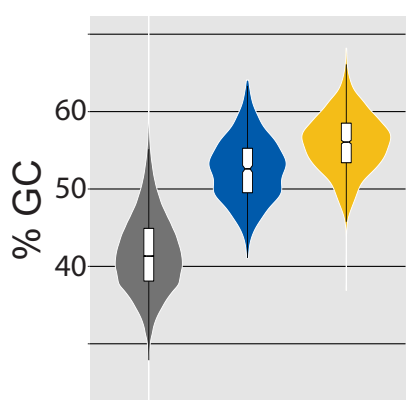

devTSS-NMI

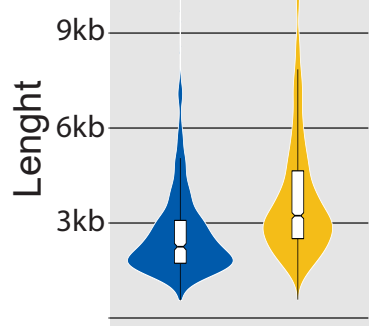

C

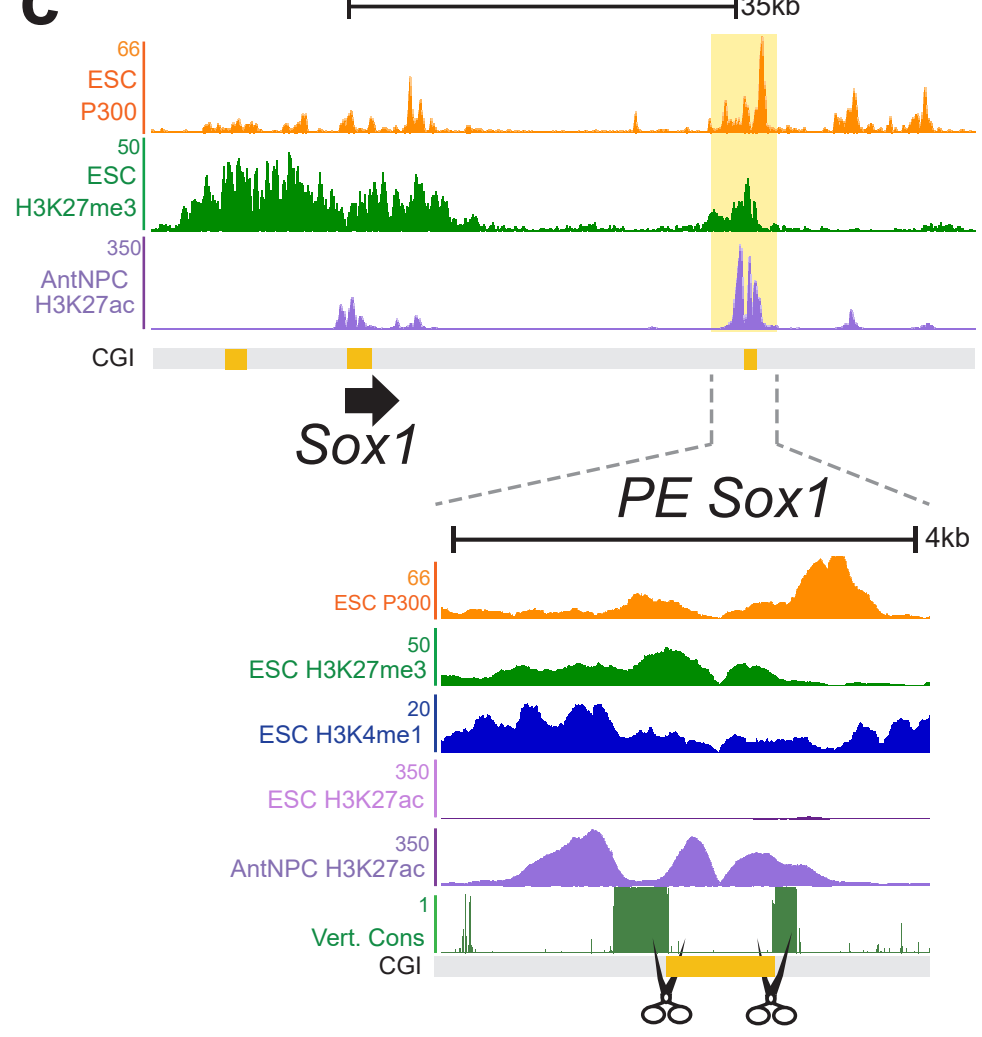

d

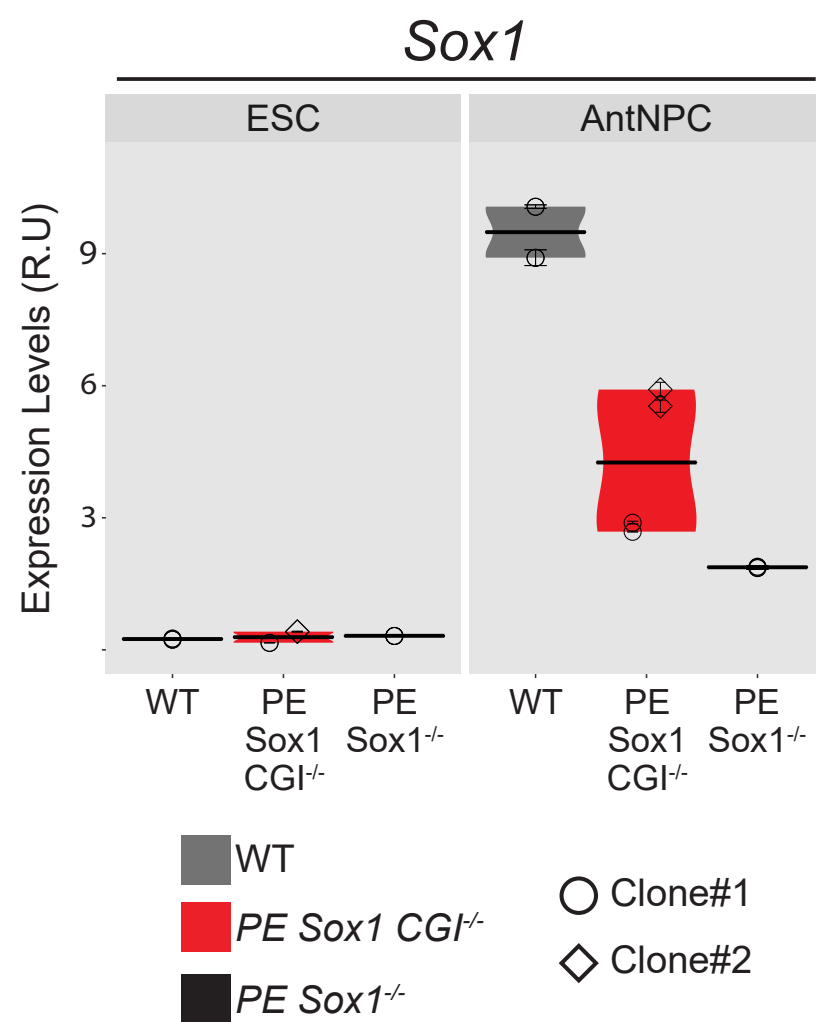


Fig. 1| Genetic properties and functional relevance of the orphan CGIs associated with PE. a, Percentage of poised enhancers (PEs) found within the indicated maximum distances $(0.25 \mathrm{~Kb}$ or $3 \mathrm{~Kb})$ to a computationally-defined CGI according to the following criteria: GC content > 50\%; Length > $200 \mathrm{bp}$; $\mathrm{CpG}$ (left panel) or a NMI identified with the Bio-CAP $\operatorname{assay}^{26}$ (right panel). PEs categorized as PE-distal correspond to those located more than 10 $\mathrm{Kb}$ away from their closest gene. b, Comparison of the $\mathrm{CpG}$ percentage, observed/expected CpG ratio, GC percentage and sequence length between random regions (see Methods), NMIs associated with poised enhancers (PE-NMI; blue) and NMIs associated with the TSS of developmental genes (devTSS-NMI; yellow; see Methods). On top of each plot, the asterisks indicate P-values calculated using unpaired Wilcoxon tests with Bonferroni correction for multiple testing $\left(* *=\right.$ p.val $<1 \mathrm{e}^{-10} ; *$ p.val $\left.<0.05\right)$; the numbers in black indicate the median fold changes between the indicated groups; the green numbers indicate non-negligible Cliff Delta effect sizes. c, The upper panel shows ChIP-seq data ${ }^{9}$ from mESC (P300 and H3K27me3) and AntNPC (H3K27ac) at the Soxl locus. The PE Soxl(+35) is highlighted in yellow. The lower panel shows a close-up view of the PE Soxl(+35) with additional epigenomic and genomic data. The represented CGIs correspond to those computationally defined in the UCSC browser according to the following criteria: GC content > 50\%; Length > 200 bp; $\mathrm{CpG}$ Observed to expected ratio > 0.6. Vert. Cons.= vertebrate PhastCons. d, The expression of Soxl was investigated by RT-qPCR in mESCs (left panel) and AntNPC (right panel) that were either WT (grey), homozygous for a deletion of the CGI associated to the PE Soxl(+35) (PE Soxl CGI ${ }^{-/}$; red) or homozygous for the complete PE Soxl(+35) deletion ${ }^{9}$ (PE Soxl $1^{-/-}$; black). Two different PE Soxl CGI ${ }^{-/} \mathrm{mESC}$ clones (circles and diamonds) and one PE Soxl ${ }^{-/}$clone were studied. For each mESC clonal line, two technical replicates of the AntNPC differentiation were performed. The plotted expression values of each clone correspond to the average and standard deviation (error bars) from three RT-qPCR technical replicates. Expression values were normalized to two housekeeping genes (Eefla and Hprt). The results of an independent biological replicate for this experiment are shown in Extended Data Fig. 2c. 
deletion of the oCGI reduced Soxl induction by $>2$-fold (compared to $>4$-fold reduction in cells with the full PE deletion) (Fig. 1d; Extended Data Fig. 2c). These findings suggest that, rather than protecting PEs from a premature activation through the recruitment of PcG, oCGIs might positively influence the cis-activation capacity of PEs.

\section{Dissection of the regulatory logic of PEs using a genetic engineering approach}

Loss-of-function approaches based on genetic deletions have several limitations in order to conclusively assess the function of oCGIs within PEs: (i) oCGIs can be difficult to delete individually, as they frequently overlap with the nearby TFBS (Extended Data Fig. 3a); (ii) the deletion of the oCGI can affect PE activity by altering the distance between TFBS $^{29}$; (iii) PE target genes typically display complex regulatory landscapes in which multiple enhancers might control gene expression ${ }^{30}$, thus potentially masking the regulatory function of individual oCGIs; (iv) experiments based on the complete loss of CGI-bound proteins (e.g. PcG, Trithorax-group proteins $(\mathrm{TrxG})^{31}$ ) can elicit global transcriptional, epigenomic and/or topological changes in mESC and, thus, indirectly alter PE loci.

To systematically dissect the contribution of oCGIs to the regulatory function of PEs, we designed a genetic engineering approach to generate $\mathrm{mESC}$ in which the components of selected PEs can be modularly inserted (i.e. TFBS, oCGI or TFBS+oCGI) into a fixed genomic location (Fig. 2a). We reasoned that by selecting insertion sites located within TADs containing developmental genes not expressed in ESC or AntNPC and, thus, without active enhancers in these cell types, any changes in the expression of the selected genes could be attributed solely to the inserted PE sequences. Furthermore, by inserting the same PE components within TADs containing developmental genes with different types of promoters (e.g. CpG-rich or CpG-poor), we could interrogate whether gene promoters differ in their responsiveness to PEs. To implement this approach, we used a CRISPR-Cas9 knock-in system $^{32}$ to initially insert the PE Soxl(+35) components (i.e. PE Soxl(+35)TFBS (465bp), PE Sox1(+35)CGI (893bp) or PE Sox1(+35)TFBS+CGI (1370bp)) approximately $100 \mathrm{~Kb}$ downstream of Gata6 (Fig. 2a), a gene with multiple CGIs around its promoter region and lowly expressed in both ESC and AntNPC (0.4 and 0.06 FPKMs, respectively $\left.{ }^{9}\right)$. The selected insertion site was not conserved and did not overlap or was close to any CTCF binding site, thus minimizing the risk of disrupting a regulatory element. Using this strategy, we successfully established two homozygous mESCs clones for each of the PE Soxl $(+35)$ insertions described above (Extended Data Fig. 3b). Next, we measured Gata6 expression in the previous mESC lines as well as upon their differentiation into AntNPC. In 
2 bioRxiv preprih

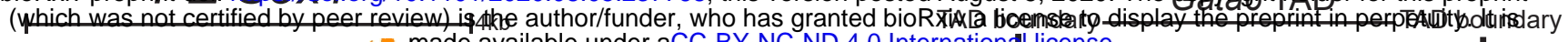

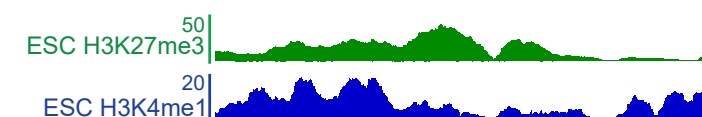

ESC H3K27ac
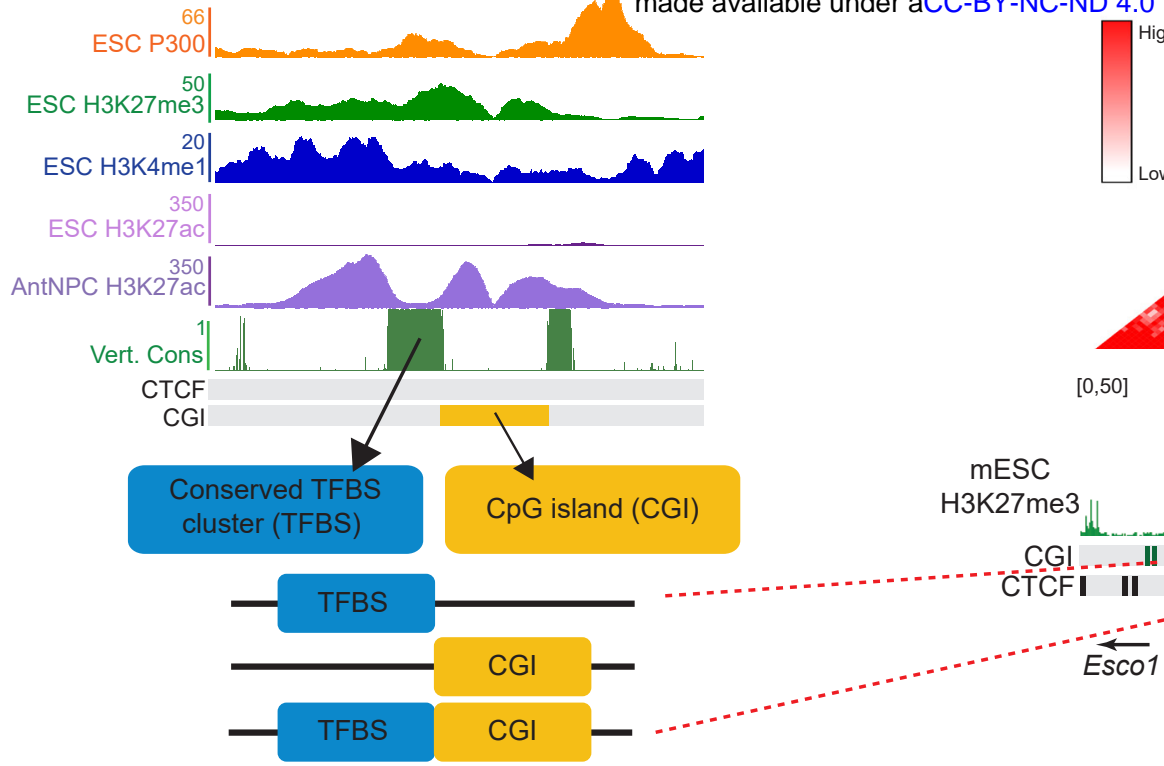

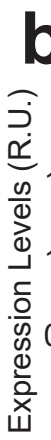

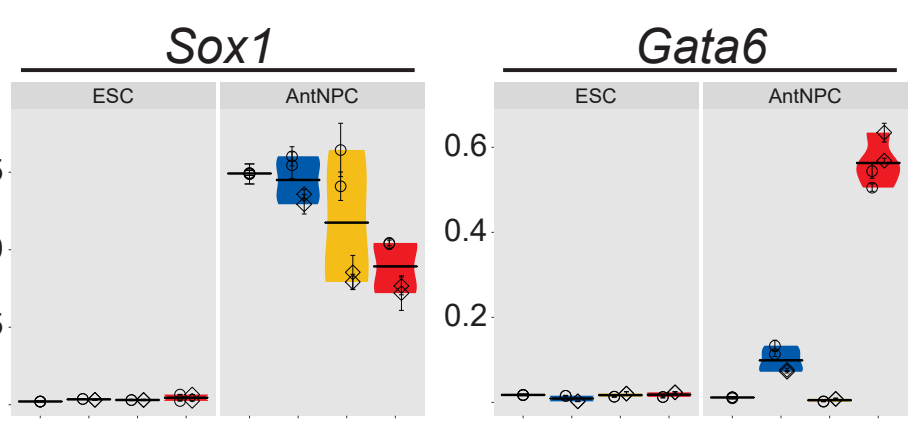

C
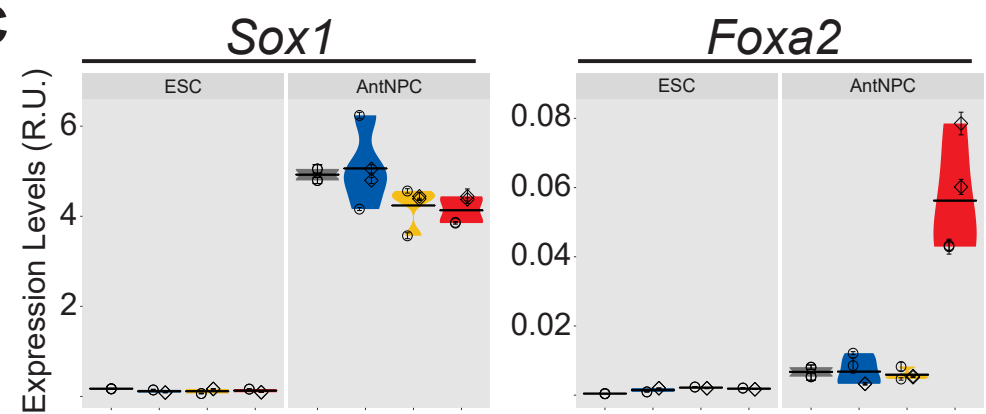

Clone\#1 $\diamond$ Clone\#2

WT TFBS CGI TFBS+CGI

d

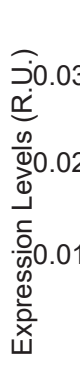
$f$

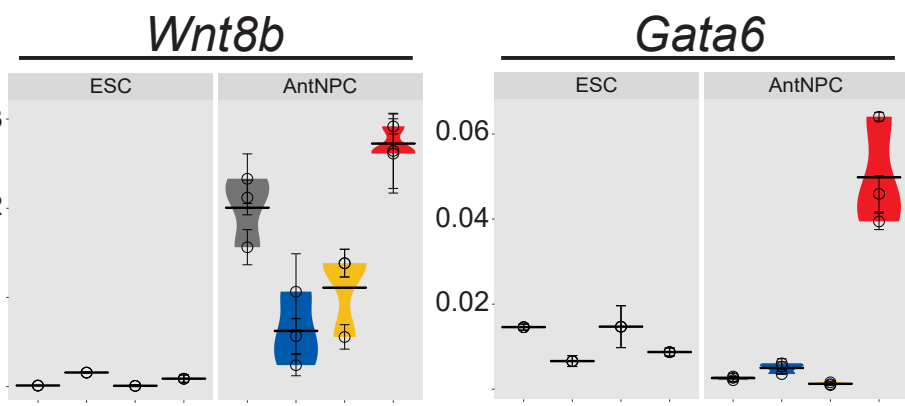

e

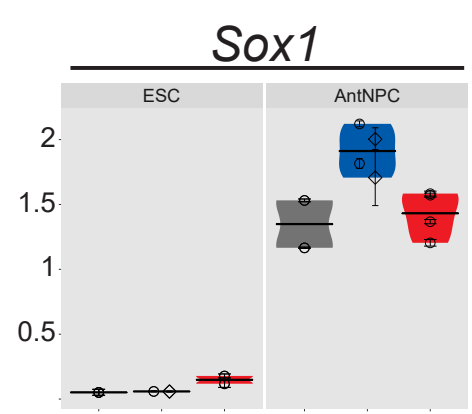

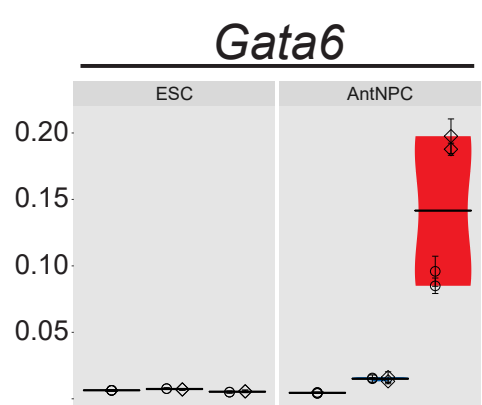

AntNPC
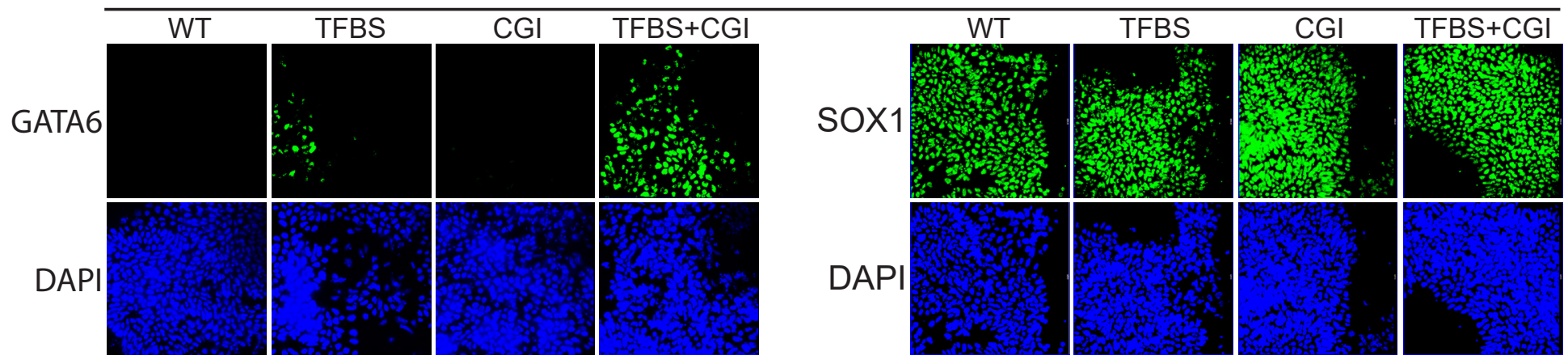
Fig. 2| Modular engineering of PEs reveals major regulatory functions for orphan CGIs. a, Strategy used to insert the PE Soxl(+35) components into the Gata6-TAD. The upper left panel shows a close-up view of the epigenomic and genetic features of the PE Soxl $(+35)$ (Vert. Cons. $=$ vertebrate PhastCons). The represented CGI was computationally defined according to the following criteria: GC content > 50\%; Length > $200 \mathrm{bp}$; CpG Observed to expected ratio $>0.6$. The lower left panel shows the three combinations of PE Soxl $(+35)$ modules (i.e. (i) PE Soxl(+35)TFBS; (ii) PE Soxl(+35)CGI; (iii) PE Sox1(+35)TFBS\&CGI) inserted into the Gata6 TAD. The right panel shows the TAD in which Gata6 is included (i.e. Gata6-TAD) according to publically available Hi-C data ${ }^{33,34}$; TAD boundaries are denoted with dotted lines; H3K27me3 ChIP-seq signals in mESC are shown in green ${ }^{9}$; CTCF binding sites in $\mathrm{ESC}^{35}$ are shown as black rectangles; CGIs are indicated as green rectangles; the red triangle indicates the integration site of the PE Soxl(+35) modules approximately $100 \mathrm{~Kb}$ downstream of Gata6. b-e, The expression of Gata6 (b,d and e), Foxa2 (c), Soxl (b, $c$ and e) and Wnt8b (c) was measured by RT-qPCR in mESCs (left panels) and AntNPC (right panels) that were either WT (grey) or homozygous for the insertions of the different PE Sox $1(+35)$ (b and c) or $P E W n t 8 b(+21)$ (d) modules (i.e. TFBS (blue), CGI (yellow), TFBS+CGI (red)). In (e), PE Sox1(+35)TFBS was inserted alone (blue) or in combination with an artificial CGI (red) into the Gata6-TAD. For the cells with the PE module insertions, two different clonal cell lines (circles and diamonds) were studied in each case. For each cell line, two technical replicates of the AntNPC differentiation were performed. The plotted expression values for each clone correspond to the average and standard deviation (error bars) from three RT-qPCR technical replicates. Expression values were normalized to two housekeeping genes (Eefla and $H p r t$ ). The results of an independent biological replicate for each experiment are shown in Extended Data Fig. 3-5. f, The expression patterns of the GATA6 (left panel) and SOX1 (right panel) proteins were investigated by immunofluorescence in AntNPCs that were either WT or homozygous for the insertion of the different PE Soxl(+35) modules (i.e. TFBS, CGI, TFBS+CGI) in the Gata6-TAD. Nuclei were stained with DAPI. 
undifferentiated ESC, we found that none of the engineered PE Sox1 $(+35)$ combinations affected Gata6 expression (Fig. 2b and Extended Data Fig. 3c). Strikingly, upon differentiation into AntNPC, Gata6 was strongly induced in cells with the $P E$ Soxl(+35)TFBS+CGI insertion ( $\sim 50$-fold $v s$ WT). In contrast, cells with the $P E$ Sox1(+35)TFBS insert displayed considerably milder Gata6 induction ( 7-fold vs WT), while the insertion of the PE Soxl(+35)CGI had not effect on Gata6 expression (Fig. 2b and Extended Data Fig. 3c). Therefore, these initial results suggest that, although the oCGI do not have cis-regulatory activity on their own, they can dramatically boost the regulatory activity of their nearby TFBS.

\section{oCGIs boost the regulatory activity of PES}

To evaluate whether the previous observations could be generalized, we used the same genetic engineering approach to generate two additional group of transgenic ESC lines: (i) the same PE Soxl(+35) components were inserted within the Foxa2-TAD $(\sim 100 \mathrm{~Kb}$ downstream of the Foxa2 TSS, which also contains several CGIs and is inactive in ESC and AntNPC); (ii) the $P E \quad W n t 8 b(+21)^{9}$ components (i.e. PE Wnt8b(+21)TFBS, $P E$ $W n t 8 b(+21) C G I$ and $P E W n t 8 b(+21) T F B S+C G I)$ were inserted within the Gata6-TAD $(\sim 100$ $\mathrm{Kb}$ downstream of the Gata6-TSS) (Extended Data Fig. 4a-d). Importantly, in both groups of cell lines we again observed that the TFBS+CGI combinations were able to strongly boost the TFBS cis-regulatory activity in AntNPC (13- and 19-fold in comparison with WT for Foxa2 and Gata6, respectively; Fig. 2c,d and Extended Data Fig. 4e,f); while the TFBS or the oCGI alone lead to either no or minor gene inductions (Fig. 2c,d and Extended Data Fig. 4e,f). Next, we also wanted to investigate whether the boosting capacity of the oCGI could be attributed to other type of regulatory information beyond their CpG-richness (e.g. binding sites for TFs). For this purpose, we designed an artificial CGI ( $\mathrm{aCGI}^{36}$; see Methods) and inserted it together with the PE Soxl(+35)TFBS at the Gata6-TAD $(P E$ Soxl(+35)TFBS $+a C G I$; Extended Data Fig. 5a,b). Notably, RT-qPCR experiments in AntNPC showed that Soxl(+35)TFBS+aCGI cells expressed considerably higher levels of Gata6 than Sox1(+35)TFBS cells (Fig. 2e and Extended Data Fig. 5c), suggesting that the CpG-richness of oCGIs is sufficient to boost the regulatory activity of PEs.

The boosting properties of oCGIs might be attributed to a premature induction of the target gene, an increase in the number of cells in which the target gene becomes induced and/or an increase in the expression levels within individual cells. To address this, we focused on those 
cell lines containing the different $P E \operatorname{Soxl}(+35)$ components inserted within the Gata6-TAD. Upon differentiation of the Soxl(+35)TFBS+CGI ESC into AntNPC, Gata6 did not become significantly induced until Day4, thus perfectly matching the expression dynamics of Soxl (the endogenous target of PE Soxl(+35)) and arguing against premature target gene induction due to the presence of the oCGI (Extended Data Fig. 6). Next, we performed immunofluorescence assays to visualize GATA6 and SOX1 protein levels in both mESC and AntNPC. In agreement with its widespread expression in neural progenitors ${ }^{37}$, SOX1 became strongly and homogenously induced in AntNPC derived from all the evaluated cell lines (Fig. 2f). Notably, GATA6 was also induced in most of the AntNPC derived from PE Soxl(+35)TFBS+CGI mESC (Fig. 2f), thus closely recapitulating the SOX1 expression pattern. In contrast, the PE Soxl(+35)TFBS insert resulted in a considerably noisier and more heterogeneous expression of GATA6, while no GATA6 could be detected in $P E$ Sox1(+35)CGI cells. These results suggest that, during pluripotent cell differentiation, oCGIs increase the number of cells in which the PE target genes get induced, thus potentially leading to increased gene expression precision ${ }^{38}$.

Taken together, we conclude that oCGIs are an essential component of PEs that might endow them with privileged regulatory properties (e.g. gene expression precision). Notably, our data also suggest that developmental genes with pCGI (e.g. Gata6 and Foxa2) are intrinsically responsive to PEs.

\section{The boosting capacity of oCGIs does not involve the local activation of PEs}

Having identified oCGIs as boosters of PE regulatory activity, we then investigated the mechanisms whereby the oCGI could exert such function, focusing on the mESC lines in which the PE Soxl(+35) components were inserted within the Gata6-TAD. CGIs are typically devoid of $\mathrm{CpG}$ methylation and display low nucleosomal density, which might provide a chromatin environment permissive for TF binding and transcription initiation ${ }^{39-43}$. However, these epigenetic features have been mostly investigated in the context of pCGI. To investigate whether oCGIs can similarly influence the chromatin environment of PEs, we first performed bisulfite sequencing experiments in $P E$ Soxl $(+35) T F B S+C G I$ and $P E$ Soxl(+35)TFBS cells to measure CpG methylation within the TFBS module. Remarkably, in undifferentiated mESC, the TFBS sequences acquired intermediate $\mathrm{CpG}$ methylation levels when inserted alone, while becoming completely unmethylated when combined with the oCGI (Fig. 3a and Extended Data Fig. 7a). Next, we performed FAIRE assays to investigate whether the oCGI could similarly impact chromatin accessibility within the engineered PEs 
Fig. 3| Characterization of the epigenetic, topological and regulatory features of the $P E$ Sox1 $(+35)$ modules engineered within the Gata6-TAD. a, Bisulfite sequencing analyses during mESC (Day0) to AntNPC (Day5) differentiation of cell lines with the $P E$ Soxl(+35)TFBS or PE Soxl(+35)TFBS+CGI modules inserted in the Gata6-TAD. DNA methylation levels were measured using a forward bisulfite primer upstream of the insertion site and a reverse primer inside the TFBS module (see Methods). b, H3K27ac and P300 levels at the endogenous PE Soxl(+35), the Gata6-TAD insertion site (primer pairs P1 and P2) and the Gata6 promoter were measured by ChIP-qPCR in mESCs (left panels) and AntNPC (right panels) that were either WT (gray) or homozygous for the insertions of the different PE Soxl(+35) modules (i.e. TFBS (blue), CGI (yellow), TFBS+CGI (red)). ChIPqPCR signals were normalized against two negative control regions (Supplementary Data 1). Error bars correspond to standard deviations from technical triplicates. The location of the primers $\mathrm{P} 1$ and $\mathrm{P} 2$ around the Gata6 TAD insertion site is represented as red arrows in the lower diagram. c, eRNA levels at the endogenous $P E$ Soxl $(+35)$ and the Gata6-TAD insertion site (primers P1 and P2) were measured by RT-qPCR in mESCs (left panels) and AntNPC (right panels) that were either WT (gray) or homozygous for the insertions of the different PE Soxl(+35) modules (i.e. TFBS (blue), CGI (yellow), TFBS+CGI (red)). The plotted expression values correspond to the average and standard deviation (error bars) from three RT-qPCR technical replicates. Expression values were normalized to two housekeeping genes (Eefla and Hprt). d,e, RNAP2 and MED1 (d) or H3K27me3 and SUZ12 (e) levels were measured by ChIP-qPCR as described in (b). f, 4C-seq experiments were performed using the Gata6-TAD insertion site (upper panels) or the Gata6 promoter (lower panels) as viewpoints in mESCs that were either WT (black) or homozygous for the insertions of the different PE Soxl(+35) modules (i.e. TFBS (blue), CGI (yellow), TFBS+CGI (red)). 
(Extended Data Fig. 7b). In contrast to the dramatic effects observed for $\mathrm{CpG}$ methylation, the oCGI only moderately increased chromatin accessibility whether inserted alone or in conjunction with the TFBS. To simultaneously measure nucleosome occupancy and $\mathrm{CpG}$ methylation levels at the inserted TFBS with single-DNA molecule resolution ${ }^{44}$, we also performed NOME-PCR assays in PE Soxl(+35)TFBS+CGI and PE Soxl(+35)TFBS mESC. These experiments confirmed that oCGIs protect nearby TFBS from CpG methylation without having a major effect on chromatin accessibility (Extended Data Fig. 7c,d). Furthermore, upon differentiation into AntNPC, the TFBS also got progressively demethylated in the $P E$ Soxl(+35)TFBS cells (Fig. 3a and Extended Data Fig. 7a), suggesting that, even in the absence of an oCGI, TFs can access and activate PEs in AntNPC ${ }^{45}$. To test this prediction, we performed ChIP-qPCR experiments to measure p300 binding and H3K27ac levels, two major hallmarks of active enhancers ${ }^{46,47}$, around the inserted PE Soxl(+35) constructs. Interestingly, these experiments showed that in AntNPC, the PEs containing the TFBS alone or together with the oCGI became strongly and similarly enriched in H3K27ac and P300 (Fig. 3b). Overall, these results indicate that the boosting capacity of the oCGI cannot be simply attributed to their local effects on the chromatin properties of PEs. Nevertheless, the CpG hypomethylation that the oCGI confer to PEs might be related to non-regulatory functions, such as preventing C-to-T mutations ${ }^{48}$.

\section{oCGIs increase the physical and functional communication between PEs and their target genes}

Another distinctive hallmark of active enhancers is the production of short bidirectional transcripts termed enhancers RNAs (eRNAs), which might be a better predictor of enhancer activity than H3K27ac ${ }^{49-52}$. Remarkably, RT-qPCR analyses in AntNPC showed a >20-fold increase in eRNA levels around the Soxl $(+35) T F B S+C G I$ insert in comparison with the $P E$ Soxl(+35)TFBS alone (Fig. 3c), thus in full agreement with the effects that these two inserts have on Gata6 expression. Moreover, additional ChIP-qPCR experiments revealed that, upon AntNPC differentiation, the Soxl(+35)TFBS+CGI insert became highly enriched in RNA Polymerase II (RNAP2) and Mediator, a protein complex that acts as a functional bridge transmitting regulatory information from enhancers to promoters ${ }^{53}$ (Fig. 3d). In contrast, the binding of these proteins to the Soxl $(+35) T F B S$ and Soxl $(+35) C G I$ inserts was either considerably weaker or undetectable, respectively (Fig. 3d). Similarly, the recruitment of RNA Pol2 and Mediator at the Gata6 promoter was also stronger in AntNPC derived from the PE Soxl(+35)TFBS+CGI mESC in comparison with the other cell lines (Fig. 3d). All 
together, these results suggest that oCGIs increase the functional communication between PEs and their target genes, resulting in elevated levels of both eRNAs and mRNAs.

In their inactive state, PEs are enriched in histone modifications (i.e. H3K27me3 and H3K4me1) and are bound by protein complexes (e.g. PcG) that have been previously implicated in the establishment of long-range chromatin interactions ${ }^{8,9,54-59}$. Therefore, we wondered if oCGIs could be implicated in the establishment of the PEs unique chromatin signature and thereby facilitate the physical communication between PEs and their target genes. To investigate this possibility, we first performed ChIPs for H3K4me1, H3K4me3 and H3K27me3 in the mESC lines containing the different PE Sox $1(+35)$ components within the Gata6-TAD (Fig. 3e and Extended Data Fig. 8a,b). H3K4me1 was weakly and similarly enriched around the PE Soxl(+35) inserts containing the TFBS with or without the oCGI, while no enrichment was observed for the oCGI insert alone (Extended Data Fig. 8a). Therefore, H3K4me1 deposition seems to be dependent on the TFBS rather than on the oCGI. On the other hand, H3K4me3 was not enriched in any of the evaluated mESC lines (Extended Data Fig. 8a), indicating that oCGIs, perhaps due to their distinct genetic features (Fig. 1), do not adopt the same chromatin state as $\mathrm{pCGI}^{36}$. Most interestingly, H3K27me3 was strongly enriched around the PE Soxl(+35) inserts containing the oCGI (Fig 3e). ChIPs for additional PcG subunits (i.e. SUZ12, CBX7 and RING1B) and associated histone modifications (i.e. H2AK119ub) further confirmed that oCGIs are sufficient for the recruitment of PcG to PEs (Fig 3e and Extended Fig 8b). Intriguingly, PRC1 recruitment (i.e. H2AK119ub, CBX7 and RING1b) was considerably stronger for the TFBS+oCGI insert than for the insert containing the oCGI alone (Extended Data Fig. 8b).

Since PcG can mediate the establishment of long-range homotypic interactions between distal PcG-bound loci ${ }^{54-58}$, we then investigated the 3D organization of the Gata6 locus in our engineered mESC lines. 4C-seq experiments using either the Gata6 promoter or the PE Soxl(+35) insertion site as viewpoints revealed that strong PE-Gata6 contacts were only established in the PE Soxl(+35)TFBS+CGI cells (Fig. 3f). The lack of PE-gene contacts in Sox1(+35)CGI ESC can be attributed to the weaker recruitment of PRC1 to the PE in these cells (Extended Data Fig. 8b), since long-range interactions between PcG-bound loci are considered to be mediated by $\mathrm{PRC} 1^{60-63}$. Furthermore, the strong interactions between the $P E$ Soxl(+35)TFBS+CGI insert and the Gata6 promoter were also observed upon differentiation into AntNPC (Extended Data Fig. 8c). In AntNPC, the TFBS+CGI insert lost H3K27me3 and PRC2, thus mirroring the concomitant and strong H3K27ac gains observed in those same 
cells (Fig. 3e). Therefore, different factors might contribute to the physical communication between PEs and their target genes promoters before and after PE activation.

Overall, our data strongly suggest that, rather than locally facilitating PE activation (i.e. p300 recruitment and $\mathrm{H} 3 \mathrm{~K} 27 \mathrm{ac}$ deposition), oCGIs boost the cis-regulatory activity of PEs by bringing genes and enhancers into close spatial proximity and, thus, increasing their functional communication.

\section{Genes with CpG-poor promoters do not show long-range responsiveness to PES}

All the developmental genes used as PE targets in our genetic engineering approach (Fig. 2) have CpG-rich promoters and are bound by PcG in ESC. Therefore, the high responsiveness of these genes to the PEs could depend not only on the presence of an oCGI within the PE (Fig. 2), but also on CGIs located at the target gene promoters. These CGIs could impose homotypic chromatin states to both PEs and promoters, thus facilitating their long-range physical and functional communication ${ }^{64}$. To test this hypothesis, we inserted the $P E$ Soxl(+35) components (i.e. PE Soxl(+35)TFBS, PE Soxl(+35)CGI or PE Soxl(+35)TFBS+CGI) into the Grial-TAD, approximately $100 \mathrm{~Kb}$ upstream of the Grial TSS (Fig 4a and Extended Data Fig. 9a). Similarly to Gata6 and Foxa2, Grial is not expressed in either mESC or AntNPC ( 0 and 0.04 FPKMs, respectively $\left.{ }^{9}\right)$. However, and in contrast to these genes, the Grial promoter does not contain CGIs and is not bound by PcG but fully DNA methylated instead. Remarkably, RT-qPCR analysis of these Grial-TAD cell lines showed that, upon AntNPC differentiation, none of the PE Soxl(+35) inserts was able to induce Grial expression (Fig. 4b and Extended Data Fig 9b).

To gain further mechanistic insights into the lack of responsiveness of Grial to the PE Sox1(+35), we then measured DNA methylation, H3K27ac, P300, RNAP2, MED1 and eRNA levels around the inserted PE Soxl(+35) constructs. Similarly to what we observed within the Gata6-TAD, the TFBS became demethylated in ESC, albeit partially, when combined with the oCGI (Extended Data Fig. 9c). Nevertheless, upon differentiation into AntNPC, the Soxl(+35)TFBS+CGI and Soxl(+35)TFBS inserts became strongly and similarly enriched in H3K27ac and P300 (Fig. 4c). Therefore, as within the Gata6-TAD, the TFBS module was sufficient for the local activation of the PE. However, and in contrast to what we observed in the Gata6-TAD, we did not detect eRNA production by any of the PE Sox1 $(+35)$ inserts (Fig. 4d). Congruently, the recruitment of RNAP2 and MED1 to the PE Sox1(+35) was weak regardless of whether the TFBS were alone or together with the oCGI. (Extended Data Fig. 9d). Furthermore, the previous transcriptional regulators were not recruited to the Grial 


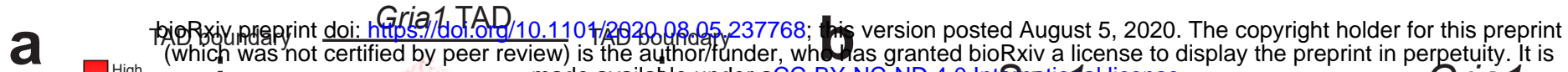
made available under aCC-BY-NC-ND 4.0 Int \& 8 xioral license. Gria 1

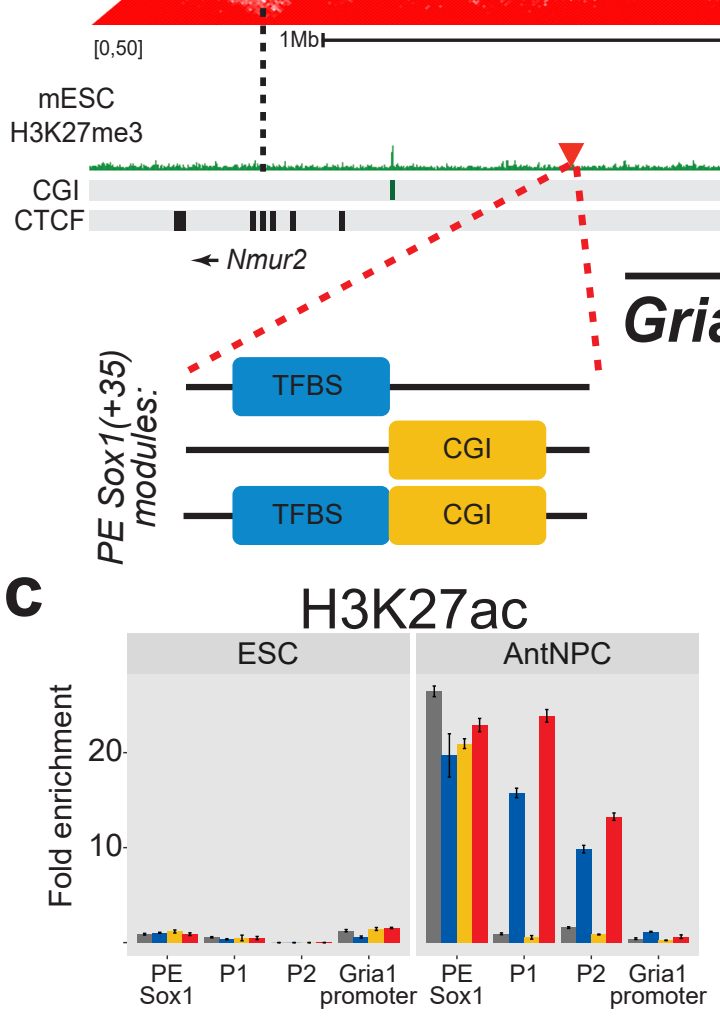

$\mathbf{e}$
0
0
0
$\mathbf{g}$
$\mathbf{g}$
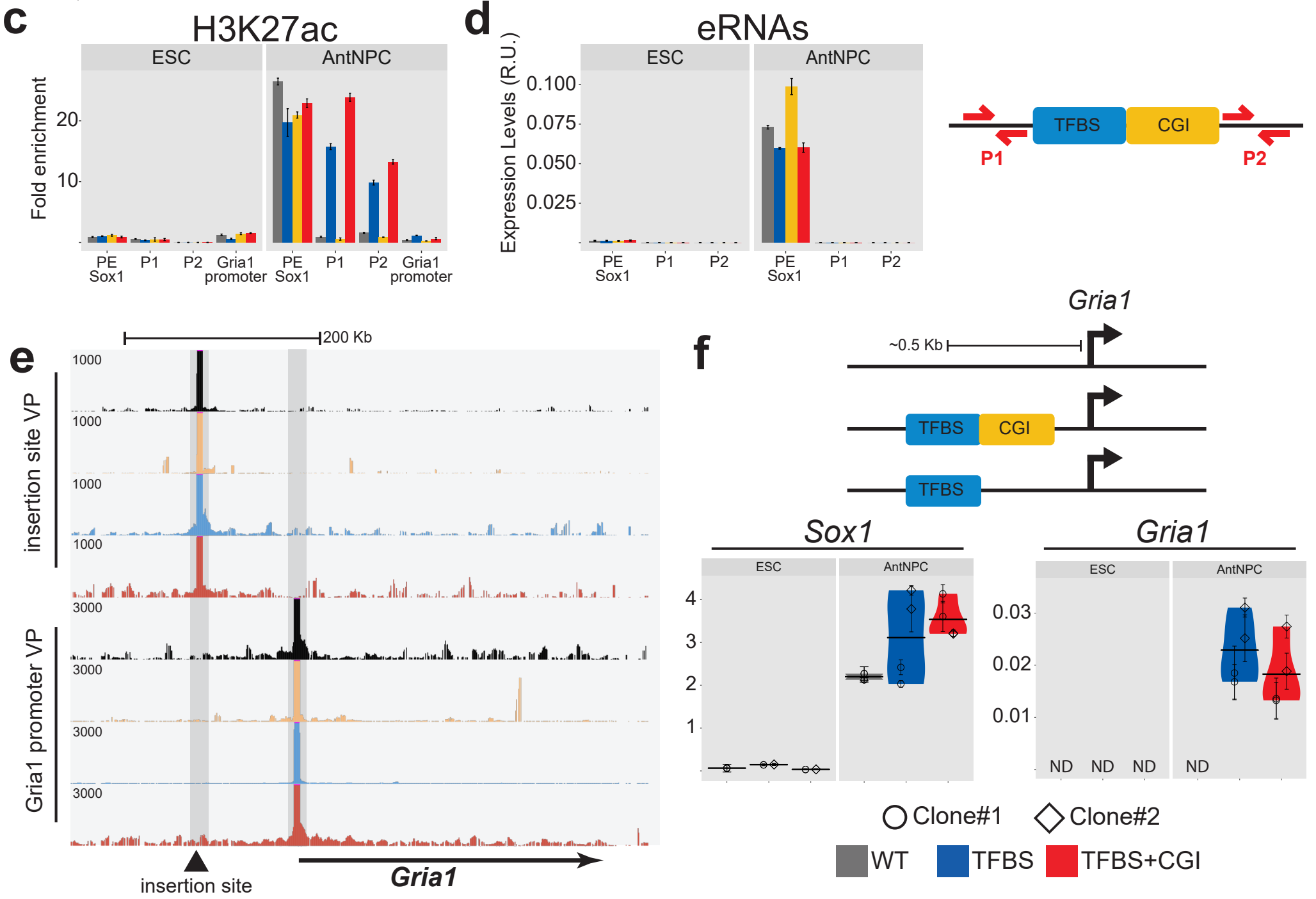

g
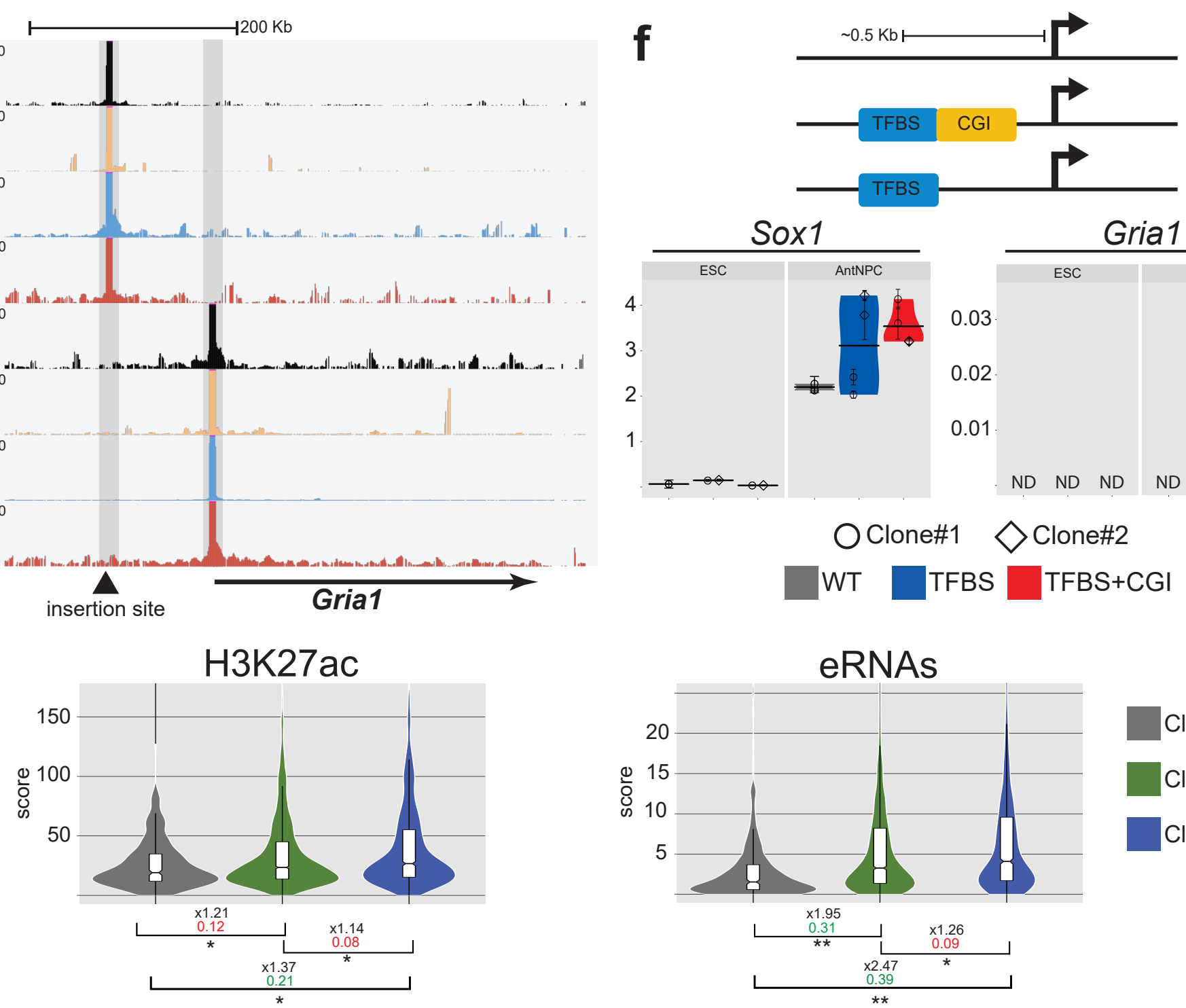

Gria1

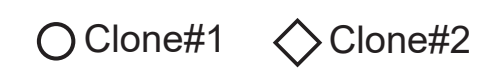

WT TFBS TFBS+CGI

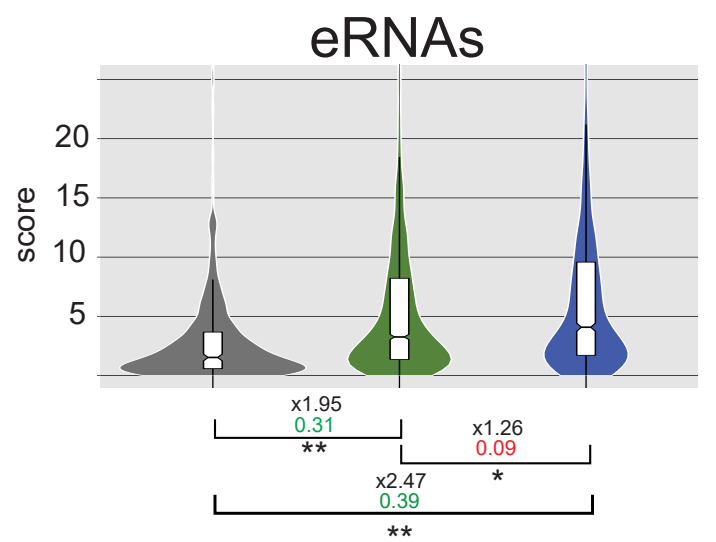

Class I

Class II

Class III 
Fig. 4| Orphan CGI do not exert their long-range boosting effects over genes with CpGpoor promoters. a, Strategy used to insert the PE Soxl $(+35)$ components into the GrialTAD. The lower panel shows the three different combinations of PE modules (i.e. (i) $P E$ Soxl(+35)TFBS; (ii) PE Soxl(+35)CGI; (iii) PE Soxl(+35)TFBS\&CGI) inserted into the Grial-TAD. The upper panel shows the TAD in which Grial is included (i.e. Grial-TAD) according to publically available $\mathrm{Hi}-\mathrm{C}$ data ${ }^{33,65}$; TAD boundaries are denoted with dotted lines; H3K27me3 ChIP-seq signals in mESC are shown in green ${ }^{9}$; CTCF binding sites in $\mathrm{ESC}^{35}$ are shown as black rectangles; CGIs are indicated as green rectangles; the red triangle indicates the integration site of the PE Soxl $(+35)$ modules, approximately $100 \mathrm{~Kb}$ upstream of Grial. b, The expression of Grial and Soxl was measured by RT-qPCR in mESCs (left panels) and AntNPC (right panels) that were either WT (grey) or homozygous for the insertions of the different PE Soxl(+35) modules (TFBS (blue), CGI (yellow), TFBS+CGI (red)). Grial expression was also measured in mouse embryonic brain to illustrate the quality of the RT-qPCR primers. For the cells with the PE module insertions, two different clonal lines (circles and diamonds) were studied in each case. For each cell line, two technical replicates of the AntNPC differentiation were performed. The plotted expression values for each clone correspond to the average and standard deviation (error bars) from three RT-qPCR technical replicates. Expression values were normalized to two housekeeping genes (Eefla and $H p r t$ ). The results of an independent biological replicate for each experiment are shown in Extended Data Fig. 9b. c, H3K27ac levels at the endogenous PE Sox1(+35), the Grial TAD insertion site (primer pairs P1 and P2) and the Grial promoter were measured by ChIP-qPCR in mESCs (left panels) and AntNPC (right panels) that were either WT (grey) or homozygous for the insertions of the different PE Soxl(+35) modules (i.e. TFBS (blue), CGI (yellow), TFBS+CGI (red)). ChIP-qPCR signals were normalized against two negative control regions (Supplementary Data 1). Error bars correspond to standard deviations from technical triplicates. d, eRNAs levels at the endogenous PE Soxl(+35) and the Grial-TAD insertion site (primer pair P1 and P2) were measured by RT-qPCR in mESCs (left panels) and AntNPC (right panels) that were either WT (grey) or homozygous for the insertions of the different Soxl(+35) modules (i.e. TFBS (blue), CGI (yellow), TFBS+CGI (red)). The plotted expression values correspond to the average and standard deviation (error bars) from three RT-qPCR technical replicates. Expression values were normalized to two housekeeping genes (Eefla and Hprt). e, 4C-seq experiments were performed using the Grial-TAD insertion site (upper panels) or the Grial promoter (lower panels) as viewpoints in mESCs (left panels) and AntNPC (right panels) that were either WT (black) or homozygous for the insertions of the 
different PE Soxl(+35) modules (i.e. TFBS (blue), CGI (yellow), TFBS+CGI (red)). f, The expression of Grial and Soxl was measured by RT-qPCR in mESCs (left panels) and AntNPC (right panels) that were either WT or homozygous for the indicated PE Soxl $(+35)$ modules (TFBS (blue), TFBS+CGI (red)), which were inserted immediately upstream of the Grial TSS. For the cells with the PE module insertions, two different clonal lines (circles and diamonds) were studied in each case. For each cell line, two technical replicates of the AntNPC differentiation were performed. The plotted expression values for each clone correspond to the average and standard deviation (error bars) from three RT-qPCR technical replicates. Expression values were normalized to two housekeeping genes (Eefla and Hprt). The results of an independent biological replicate for each experiment are shown in Extended Data Fig. 10c. g, Active enhancers identified in mESC based on the presence of distal H3K27ac peaks (see Methods) were classified into three categories: Class I correspond to active enhancers located in TADs containing only poorly expressed genes $(<0.5$ FPKM); Class II correspond to active enhancers located in a TAD with at least one gene with expression levels of >10 FPKM; Class III correspond to active enhancers whose closest genes in their same TAD have expression levels of $>10$ FPKM. The violin plots show the H3K27ac (left) and eRNA (right) levels for each of these active enhancer categories in mESC. On the bottom of each plot, the asterisks indicate P-values calculated using unpaired Wilcoxon tests with Bonferroni correction for multiple testing $\left(* *=\right.$ p.val $<1 \mathrm{e}^{-10}$; $*$ p.val $\left.<0.05\right)$; the numbers in black indicate the median fold-changes between the indicated groups; the coloured numbers correspond to Cliff Delta effect sizes: negligible (red) and non-negligible (green). The H3K27ac ChIP-seq data was obtained from ${ }^{9}$ and the PRO-seq data to measured eRNA levels was obtained from ${ }^{66}$. 
promoter upon differentiation of any of the Grial-TAD ESC lines, thus in full agreement with the lack of Grial induction observed in those cells (Extended Data Fig. 9d).

Together with our experiments within the Gata6-TAD, these results show that developmental genes with $\mathrm{CpG}$-rich promoters are particularly responsive to PEs.

\section{Uncoupling of H3K27ac and eRNA transcription during enhancer activation}

The previous genetic engineering experiments within the Gata6 and Grial-TAD revealed that H3K27ac and eRNA production can be uncoupled from each other and thus signify different steps during PE activation (Fig. 3b,c and Fig. 4c,d). Namely, the accumulation of H3K27ac occurs as PEs become locally activated, while the production of eRNAs, which is coupled to gene transcription, indicates the functional activation of the PEs. Although our findings are in agreement with the original characterization of eRNAs, in which it was reported that eRNA synthesis requires the presence of a target promoter $^{49}$, several recent studies have proposed that enhancers and promoters represent independent transcriptional units ${ }^{67,68}$. To assess whether our observations can be generalized, we compared eRNA production between three classes of active enhancers using nascent transcription and epigenomic data generated in ESC $^{66,69}$ : (I) enhancers located in TADs containing only poorly expressed genes; (II) enhancers located in a TAD with at least one gene with high expression levels; (III) enhancers whose closest gene within the same TAD has high expression levels (see Methods). Interestingly, class I enhancers showed $\sim 2$ and 2.5-fold lower eRNA levels than Class II and Class III enhancers, respectively (Fig. 4g), while H3K27ac levels were similar among the three enhancer groups. The differences in eRNA expression between enhancer classes were maintained after correcting for $\mathrm{H} 3 \mathrm{~K} 27 \mathrm{ac}$ levels or when using independent transcriptional and epigenomic data sets (Extended Data Fig. 11a-c). Although there were some Class I enhancers for which we observed detectable eRNA levels, our results suggest that enhancer and gene transcription are frequently coupled and mutually dependent on enhancer-promoter interactions ${ }^{49,50}$.

\section{oCGIs act as long-range regulatory boosters of PES}

The engineering experiments within the Gata6-TAD suggest that the responsiveness to PEs involves the physical proximity between PEs and their target genes, which in ESC is likely to be mediated by PcG present at both PEs and promoter regions ${ }^{9,60-62,70}$ (Fig. 3f). ChIP 
experiments in the Grial-TAD ESC lines revealed that, as observed for the Gata6-TAD (Fig. 3e), PcG were recruited to the PE Soxl $(+35)$ inserts containing an oCGI (Extended Data Fig. 9e). However, 4C-seq analyses in these cells showed that none of the inserted PE Soxl $(+35)$ constructs was able to significantly interact with the Grial promoter (Fig. 4e), which, as stated above, does not contain any endogenous pCGI and is not boud by PcG. These results further suggest that the main regulatory function of oCGIs is to facilitate the establishment of long-range PE-gene contacts. To test this prediction, we generated additional ESC lines in which the PE Soxl(+35)TFBS or PE Soxl(+35)TFBS\&CGI constructs were integrated 380 bp upstream of the Grial TSS (Fig. 4f, Extended Data Fig. 10a,b). Remarkably, RT-qPCR analyses in AntNPC derived from the previous ESC lines revealed that both the $P E$ Soxl(+35)TFBS+CGI and PE Soxl(+35)TFBS inserts were able to induce Grial and that they did so with similar strength (Fig. 4f and Extended Data Fig. 10c). Therefore, the boosting capacity of oCGIs is lost when PEs are located close to gene promoters, strongly suggesting that oCGIs act as long-range boosters of PE regulatory function.

\section{The combined effects of CGIs and TAD boundaries enable the specific induction of PE target genes}

Our data suggest that, in addition to TAD boundaries, the interactions between PE-associated oCGI and pCGI proximal to developmental genes represent an important regulatory layer that ensures that gene expression programs are specifically implemented during embryogenesis. To test this prediction, we decided to genetically engineer the Six3/Six2 locus due to the following reasons (Fig. 5a): (i) Six3 and Six2 are next to each other, yet they are contained within two neighbouring TADs separated by a conserved TAD boundary ${ }^{71,72}$; (ii) Six3 and Six2 display mutually exclusive expression patterns during embryogenesis (e.g. Six 3 in brain; Six2 in facial mesenchyme) ${ }^{71}$; (iii) the Six3-TAD contains a PE (i.e. PE Six3(-133)) that controls the induction of Six3 in AntNPC without any effects on $\operatorname{Six}^{9}$; (iv) in $\mathrm{mESC}$, the PE Six3(-133) strongly interacts with Six3 but not with $\operatorname{Six} 2^{9}$ although both genes contain multiple pCGI. Taking all this information into account, we generated mESC with two different genomic rearrangements: (i) a $36 \mathrm{~Kb}$ deletion spanning the Six3/Six2-TAD boundary (del36) and (ii) a $110 \mathrm{~Kb}$ inversion that places Six3 within the Six2-TAD and vice versa (inv110) (Fig. 5a and Extended Data Fig. 12a,b). Interestingly, upon differentiation into AntNPC, Six2 expression strongly increased in del36 and inv110 cells ( 12- and $\sim 35$-fold compared to WT cells, respectively); while the expression of Six3 expression was dramatically reduced in inv110 cells ( 77 -fold compared to WT cells) and mildly affected in 
Fig. 5| oCGI and TAD boundaries enable PEs to specifically induce their target genes. a, The TADs in which Six3 and Six2 are located (i.e. Six3-TAD and Six2-TAD) are shown according to publically available Hi-C data ${ }^{33,65}$; the TAD boundary separating Six 3 and Six2 is denoted with a dotted line. Below the Hi-C data, several epigenomic and genetic features of the Six3-TAD and the Six2-TAD are shown. The represented CGIs correspond to those computationally defined in the UCSC browser according to the following criteria: GC content $>50 \%$; Length > 200bp; $\mathrm{CpG}$ Observed to expected ratio > 0.6. ChIP-seq profiles for the indicated proteins and histone modifications were obtained from ${ }^{9,35}$. The rectangles indicate the location of the $36 \mathrm{~Kb}$ deletion (red) and $110 \mathrm{~Kb}$ inversion (blue) engineered in mESC. $\mathbf{b}$, The expression of Six3 (blue) and Six2 (red) was measured by RT-qPCR in mESCs and AntNPC that were either WT (left panel), homozygous for the $36 \mathrm{~Kb}$ deletion (del36; middle panel) or homozygous for the $110 \mathrm{~Kb}$ inversion (inv110; right panel). For each of the engineered structural variants, two different clonal cell lines were generated and independently differentiated into AntNPC. The plotted expression values for each clone correspond to the average and standard deviation (error bars) from three RT-qPCR technical replicates. Expression values were normalized to two housekeeping genes (Eefla and Hprt). c, Proposed model for the role of oCGI as boosters of PE regulatory activity and determinants of PE-gene compatibility. The presence of oCGI increases the physical communication of PEs with their target genes due to homotypic chromatin interactions between oCGI and promoterproximal CGI. Consequently, the oCGI can increase the number of cells and alleles in which the PEs and their target genes are in close spatial proximity (i.e. permissive regulatory topology) both during pluripotency and upon differentiation. This will ultimately result in a timely and homogenous induction of the PE target genes once the PEs become active (i.e. increase transcriptional precision). In addition, the compatibility and responsiveness between $\mathrm{PE}$ and their target genes depends on the presence of oCGI and pCGI at the PEs and the target genes, respectively. Therefore, the oCGI can increase the specificity of the PEs by enabling them to preferentially communicate with their CpG-rich target genes while still being insulated by TAD boundaries. These novel PE-gene compatibility rules can improve our ability to predict and understand the pathomechanisms of human structural variants. 
del36 cells ( 2.5-fold compared to WT cells) (Fig 5b). Furthermore, and in agreement with the previous gene expression changes, 4C-seq experiments in WT and del36 ESC showed that the deletion of the Six3/Six2 boundary resulted in increased interactions between Six2 and the PE Six3(-133) (Extended Data Fig. 12c). These results indicate that the PEs can specifically execute their regulatory functions due to the combined effects of TAD boundaries, which provide insulation, and the long-range homotypic interactions between oCGIs and pCGIs, which confer enhancer responsiveness.

\section{Discussion}

Here we show that oCGIs are an essential component of PEs that endows these distal regulatory elements with privileged regulatory properties. Namely, oCGIs boost the regulatory activity of PEs and dictate the compatibility between PEs and their target genes (Fig. 5c). Deciphering the factors that control enhancer-promoter compatibility is still a major challenge in the enhancer field that we are just starting to understand ${ }^{73,74}$. Current models of enhancer function state that insulator proteins (i.e. CTCF) demarcate TAD boundaries and restrict enhancers to act upon genes located within their same TADs ${ }^{75-78}$. Nonetheless, enhancers do not promiscuously activate all the genes present within a $\mathrm{TAD}^{20,23,79,80}$, suggesting that additional and largely unknown factors must dictate enhancer responsiveness. Using massively parallel reporter assays in Drosophila, it was recently shown that enhancer responsiveness is determined by the sequence composition of core promoters ${ }^{25,81}$. However, we now show that, at least in the context of PE loci, such responsiveness is also dependent on distal genetic elements, namely oCGIs, which allow PEs to preferentially activate CpG rich promoters (Fig. 5c). It is worth emphasizing that neither these novel enhancer-promoter compatibility rules nor the boosting capacity of oCGIs would have been uncovered with classical reporter assays in which enhancers and core promoters are placed close to each other (Fig. 4f). Therefore, genetic engineering approaches like the one presented here will be essential to dissect the mechanisms whereby distal enhancers control the expression of their target genes.

Our data suggest that the role of oCGIs as boosters of PEs regulatory function does not involve the local activation of the PEs but rather the establishment of long-range interactions with CpG-rich gene promoters (Fig. 5c). In pluripotent cells, these PE-gene interactions are mediated by PcG complexes recruited to both oCGIs and pCGIs ${ }^{8,9,82,83}$ and are likely to be dependent on the polymerization and/or phase separation properties of $\mathrm{PRC} 1^{60-62,84}$. Subsequently, PcG might keep PEs and their target genes close together during pluripotent 
cell differentiation, ensuring that, as the PEs become active, they can rapidly and uniformly induce the expression of their target genes. Then, once RNAs are produced at both PEs and their target genes, this would result in $\mathrm{PcG}$ eviction ${ }^{85}$. Although $\mathrm{PRC} 1^{63}$ might also contribute to PE-gene communication once PEs become active, additional proteins and mechanisms are likely to be involved. Interestingly, we showed that, upon PE activation, the oCGI dramatically increase the loading of Mediator and the transcriptional machinery to both PEs and their CpG-rich target genes (Fig. 5c). We speculate that, by facilitating the physical communication between genes and enhancers, the oCGI might favour the formation of phaseseparated transcriptional condensates ${ }^{86,87}$. Once PEs are already active, multivalent interactions occurring within these condensates could robustly maintain PE-gene communication $^{88}$.

Overall, we propose a model whereby the precise and specific induction of certain developmental genes is achieved through the combination of CGI-mediated long-range chromatin interactions and the insulation provided by TAD boundaries. We anticipate that this model may have important medical implications, as it could improve our ability to predict and understand the pathological consequences of human structural variation ${ }^{12}$ (Fig. 5c).

\section{Acknowledgements}

We thank the Rada-Iglesias lab members for insightful comments and critical reading of the manuscript.

\section{Funding}

Tomas Pachano is supported by a doctoral fellowship from the DAAD (Germany). Víctor Sánchez-Gaya is supported by a doctoral fellowship from the University of Cantabria (Spain). Work in the Rada-Iglesias laboratory was supported by the EMBO YIP programme, CMMC intramural funding (Germany), the German Research Foundation (DFG) (Research Grant RA 2547/2-1), "Programa STAR-Santander Universidades, Campus Cantabria Internacional de la convocatoria CEI 2015 de Campus de Excelencia Internacional” (Spain) and the Spanish Ministry of Science, Innovation and Universities (Research Grant PGC2018-095301-B-I00). The Landeira laboratory is funded by grants of the Spanish Ministry of Science and 
Innovation (BFU2016-75233-P and PID2019-108108GB-I00) and the Andalusian Regional Government (PC-0246-2017).

\section{Author Contributions}

Conceptualization: T.P., A.R-I.; Experimental investigation: T.P., M.M-F., T.E., P.R., H.G.A.; Data analysis: T.P., V.S-G.; Writing, Review \& Editing: T.P., A.R-I; Resources: S.CM.,WFJ.vI., D.L., A.R.-I.; Supervision and Funding Acquisition: A.R.-I.;

\section{Declaration of Interests}

The authors declare no competing interests.

\section{Methods}

\section{Cell lines and differentiation protocol}

mESC (E14) were cultured on gelatin-coated plates using Knock-out DMEM (KO-DMEM, Life Technologies) supplemented with 15\% FBS (Life Technologies) and LIF. These mESC lines were differentiated into AntNPC according to a previously described protocol ${ }^{89}$, with slight modifications. Briefly, mESC were plated at a density of 12.000 cells $/ \mathrm{cm}^{2}$ on gelatincoated plates and grown for three days in N2B27 medium supplemented with $10 \mathrm{ng} / \mathrm{ml} \mathrm{bFGF}$ (Life Technologies) and without serum or LIF. N2B27 medium contains: Advanced Dulbecco's Modified Eagle Medium F12 (Life Technologies) and Neurobasal medium (Life Technologies) (1:1), supplemented with 1x N2 (Life Technologies), 1x B27 (Life Technologies), 2 mM L-glutamine (Life Technologies), 40 mg/ml BSA (Life Technologies), $0.1 \mathrm{mM}$ 2-mercaptoethanol (Life Technologies)). Subsequently, cells were grown for another two days in N2B27 medium without bFgf (D3-D5). To improve the homogeneity of the differentiation, from D2-D5 the N2B27 medium was also supplemented with $5 \mathrm{mM}$ Xav939, a potent WNT inhibitor ${ }^{90}$.

\section{RNA isolation, cDNA synthesis and RT-qPCR}

Total RNA was isolated using Innuprep RNA mini kit (Analytik Jena) according to the manufacturer's instructions. cDNA was generated using ProtoScript II First Strand cDNA 
Synthesis Kit (New England Biolabs). RT-qPCRs were performed on the Light Cycler 480II (Roche) using Eeflal and Hptr as housekeeping genes. All primers used in RT-qPCR analysis are shown in Supplementary Data 1.

\section{ChIP}

ChIPs were performed as previously described ${ }^{8}$. Basically, 50 million cells for P300/RNAP2/Med1/PcG subunits ChIPs or 10 million cells for histone ChIPs were crosslinked with $1 \%$ formaldehyde for $10 \mathrm{~min}$ at room temperature (RT) and then quenched with $0,125 \mathrm{M}$ glycine for another $10 \mathrm{~min}$. Cells were then washed with PBS and resuspended sequentially in three different lysis buffers (Lysis Buffer 1: $50 \mathrm{mM}$ HEPES $140 \mathrm{mM} \mathrm{NaCl} 1$ mM EDTA 10\% glycerol 0.5\% NP-40 0.25\% TX-100, Lysis Buffer 2: $10 \mathrm{mM}$ Tris $200 \mathrm{mM}$ $\mathrm{NaCl} 1 \mathrm{mM}$ EDTA 0.5 mM EGTA, Lysis Buffer 3: $10 \mathrm{mM}$ Tris $100 \mathrm{mM} \mathrm{NaCl} 1 \mathrm{mM}$ EDTA $0.5 \mathrm{mM}$ EGTA $0.1 \%$ Na-Deoxycholate $0.5 \% \mathrm{~N}$-lauroylsarcosine) in order to isolate chromatin. Chromatin was then sonicated for 15 cycles (20s on 30s off, $25 \%$ amplitude) using an EpiShear probe sonicator (Active Motif). After sonication, samples were centrifuged at $16000 \mathrm{~g}$ during $10 \mathrm{~min}$ at $4^{\circ} \mathrm{C}$, with the supernatant representing the sonicated chromatin. Chromatin was then incubated overnight at $4^{\circ} \mathrm{C}$ with $3 \mathrm{ug}$ antibody for histones or $10 \mathrm{ug}$ antibody for the of the investigated proteins. One of the aliquots was not subject to immunoprecipitation, thus representing total input control for the ChIP reactions. Next, $50 \mathrm{ul}$ of protein $\mathrm{G}$ magnetic beads (Invitrogen) were added to the ChIP reactions and incubated for four additional hours at $4^{\circ} \mathrm{C}$. Magnetic beads were washed and chromatin eluted, followed by reversal of the crosslinking and DNA purification. The resulting ChIP and input DNAs were analysed by qPCR using two intergenic regions as negative controls (chr2:73,030,26573,030,373; chr6: 52,339,345-52,339,505). All primers used in ChIP-qPCR experiments are shown in Supplementary Data 1.

\section{Bisulfite sequencing}

Bisulfite conversion of $400 \mathrm{ng}$ of genomic DNA was performed using the EZ DNA Methylation Kit (Zymo Research). The PE Sox1(+35)TFBS or Soxl(+35)TFBS+CGI inserts in Gata6-TAD or Grial-TAD were amplified by PCR using EpiTaq polymerase (Takara Bio) and primers described in Extended Data Fig. 7a/Supplementary Data 1. PCR products were cloned into the pGEM-T vector (Promega) and sequenced with M13 reverse primer.

\section{Immunofluorescence (IF)}


Cells were rinsed with PBS and then fixed for 10 min in 3,7\% paraformaldehyde (PFA) in PBS at RT. PFA was removed and cells were rinsed with PBS. Cells were permeabilized by treating them with $0.1 \%$ Triton $\mathrm{X}-100$ for 15 minutes at RT, followed by blocking in PBS with 5\%BSA for 1 hour at RT. Incubation with primary antibodies (GATA6 (AF1700, R\&D systems) or SOX1 (AF3369, R\&D systems)) was done in blocking solution overnight (12-18 h) at $4^{\circ} \mathrm{C}$. Cells were rinsed with PBS and incubated with secondary antibodies (Life Technologies) in blocking solution for 30 minutes at RT and then rinsed again with PBS. Finally, cell nuclei were stained with DAPI (Sigma) during $10 \mathrm{~min}$ at RT and then mounted with anti-fading mounting medium (Life Technologies).

\section{C-seq}

4C-seq Circular Chromatin Conformation Capture (4C) assays were performed as previously described $^{9,91} \cdot 1 \times 10^{7} \mathrm{mESC}$ or AntNPC were crosslinked with $1 \%$ formaldehyde during 20 minutes and quenched with $0.125 \mathrm{M}$ glycine for 10 minutes. Cells were washed with PBS and resuspended in lysis buffer $(50 \mathrm{mM}$ Tris- $\mathrm{HCl} \mathrm{pH} 7.5,150 \mathrm{mM} \mathrm{NaCl}, 5 \mathrm{mM}$ EDTA, 0.5\% NP-40, 1\% TX-100 and $1 \mathrm{X}$ protease inhibitors) during 10 minutes on ice. Following centrifugation for 5 minutes at $650 \mathrm{~g}$ at $4^{\circ} \mathrm{C}$, nuclei were re-suspended in $0.5 \mathrm{~mL}$ of $1.2 \mathrm{X}$ restriction buffer with $0.3 \%$ SDS and incubated at $37^{\circ} \mathrm{C}$ and $900 \mathrm{rpm}$ for 1 hour. After that, Triton X-100 was added to a final concentration of $2 \%$ followed by 1 hour incubation at $37^{\circ} \mathrm{C}$ and $900 \mathrm{rpm}$. Afterwards, chromatin was digested overnight at $37^{\circ} \mathrm{C}$ and $900 \mathrm{rpm}$ with $400 \mathrm{U}$ of NlaIII (R0125L, NEB). NlaIII was inactivated by adding SDS to a final concentration of $1.6 \%$ and incubating the mixture for 20 minutes at $65^{\circ} \mathrm{C}$ while shaking $(900 \mathrm{rpm})$. The digested chromatin was transferred to $50 \mathrm{~mL}$ tubes and $6.125 \mathrm{~mL}$ of $1.15 \mathrm{X}$ ligation buffer (50 mM Tris-HCl pH 7.6, 10 mM MgCl2, 1 mM ATP, 1mM DTT) were added. Triton X-100 was also added to a final concentration of $1 \%$ and the resulting solution was incubated for 1 hour at $37^{\circ} \mathrm{C}$ while shaking gently. After that, digested chromatin was ligated with $100 \mathrm{U}$ of T4 DNA ligase (15224-041, Life Technologies) for 8 hours at $16^{\circ} \mathrm{C}$, followed by RNase A treatment (Peqlab) for 45 minutes at $37^{\circ} \mathrm{C}$. Subsequently, chromatin was de-crosslinked with $300 \mathrm{mg}$ of Proteinase K (Peqlab) and incubated at $65^{\circ} \mathrm{C}$ overnight. DNA was then purified by phenol/chloroform extraction followed by ethanol precipitation and re-suspension in $100 \mathrm{~mL}$ of water. At this step, the digestion and ligation efficiencies were evaluated by analysing a small fraction of the purified DNAs by agarose electrophoresis. The remaining DNA was digested with 50U of DpnII (R0543M, NEB) at $37^{\circ} \mathrm{C}$ overnight. DNA samples were then purified by phenol/chlorophorm extraction, followed by ethanol precipitation and 
resuspension in $500 \mathrm{ul}$ of $\mathrm{H}_{2} \mathrm{O}$. Afterwards, a second ligation was performed by adding $200 \mathrm{U}$ of T4 DNA Ligase into a final volume of $14 \mathrm{~mL} 1 \mathrm{X}$ Ligation Buffer and incubating overnight at $16^{\circ} \mathrm{C}$. DNA samples were subjected to another round of phenol/chlorophorm extraction and ethanol precipitation, re-suspended in $100 \mathrm{uL}$ of water and purified with a QIAgen PCR purification column (28104, QIAgen). Finally, the resulting 4C-DNA products were amplified by inverse PCR using primers located within selected PEs, which were designed as previously described $^{91}$ (Supplementary Data 1). The inverse PCRs were performed with the expand long template PCR system (11681842001, Roche) using 30 amplification cycles $\left(94^{\circ} \mathrm{C} 2 \mathrm{~min}, 30 \mathrm{x}\right.$ $\left.\left[94^{\circ} \mathrm{C} 10 \mathrm{~s}, 60^{\circ} \mathrm{C} 1 \mathrm{~min}, 68^{\circ} \mathrm{C} 3 \mathrm{~min}\right], 68^{\circ} \mathrm{C} 5 \mathrm{~min}\right) .4 \mathrm{C}$-seq libraries were then analysed by next generation sequencing. All the generated 4C-seq data will be deposited in GEO and made available upon publication.

\section{oCGI deletion using CRISPR-Cas9}

To generate the deletion of the PE Soxl(+35)CGI, pairs of sgRNAs flanking the oCGI were selected according to Benchling's CRISPR toolkit (www.benchling.com) (Supplementary Data 1). For each selected sgRNA, two oligonucleotides were synthesized (Integrated DNA Technologies) and annealed. The CRISPR-Cas9 expression vector, pX330-hCas9-longchimeric-grna-g2p (provided by Leo Kurian's laboratory), was digested with BbsI (R0539L, NEB) and gel or column purified. Pairs of annealed oligos and the digested vector were ligated overnight at $16^{\circ} \mathrm{C}$ using T4 ligase (NEB). Following transformation, the gRNA-Cas9 expression vectors were purified and sequenced to confirm that the gRNAs were correctly cloned. mESC were transfected with the pair of gRNAs-Cas9 expressing vectors using Lipofectamine according to the manufacturer protocol (Thermo Scientific). After 16 hours, puromycin selection was performed for 48 hours. Subsequently, surviving cells were isolated in 96-well plates by serial dilution and, following expansion, clones with the desired deletion were identified by PCR using the primers listed in Supplementary Data 1. Finally, the presence of the desired deletion was confirmed in the selected mESC clones by Sanger sequencing.

\section{Homology-dependent Knock-in}

Knock-In of PE modules was performed using CRISPR-Cas9 as previously described by Yao and colleagues ${ }^{32}$ with minor modifications. First, a sgRNA was designed for the insertion site of interest and cloned in a CRISPR-Cas9 expression vector as mentioned above. Then, the cassette-vector was generated by ligating: (i) 300bp homology arms flanking the insertion 
site; (ii) construct of interest; and (iii) cloning vector. The resulting cassette-vector was used as a template for amplifying the knock-in donor (left homology arm + construct + right homology arm) by PCR (Supplementary Data 2). The resulting PCR product was purified using QIAgen PCR purification columns (28104, QIAgen). mESC were transfected with the sgRNA-Cas9 expressing vector and the knock-in donor using Lipofectamine according to the manufacturer protocol (Thermo Scientific). After 16 hours, puromycin selection was performed for 48 hours. Subsequently, surviving cells were isolated in 96-well plates by serial dilution and, following expansion, clones with the desired insertions were identified by PCR using the primers listed in Supplementary Data 1. Finally, the insertion of the desired PE modules was confirmed in the selected $\mathrm{mESC}$ clones by Sanger sequencing.

\section{FAIRE (Formaldehyde-Assisted Isolation of Regulatory Elements)}

Sonicated chromatin was prepared as for ChIP and then DNA was purified as previously described $^{92}$. Briefly, chromatin was subject to three rounds of phenol/chloroform purification and the resulting DNA was purified by precipitation with sodium acetate and ethanol. The resulting FAIRE and input DNAs were analysed by qPCR using two intergenic regions as negative controls (chr2:73,030,265-73,030,373; chr6:52,339,345-52,339,505). All primers used in the FAIRE-qPCR experiment are shown in Supplementary Data 1.

\section{NOMe-PCR}

Nuclei extraction and M.CviPI treatment were performed as described previously ${ }^{93}$ with minor modifications. Basically, isolated nuclei were incubated with $200 \mathrm{U}$ of M.CviPI (NEB) for $15 \mathrm{~min}$ at $37^{\circ} \mathrm{C}$. Then, bisulfite conversion was performed using the EZ DNA Methylation Kit (Zymo Research) and the converted DNA was amplified by PCR. Finally, the PCR product were cloned into the pGEM-T vector (Promega) and sequenced with M13 reverse primer. NOMe-PCR data was analysed with the NOMePlot web app tool (http://www.landeiralab.ugr.es/soltware) ${ }^{94}$.

\section{Computational and Statistical Analyses}

\section{Analyses of q-PCR data}

For RT-qPCR, relative gene expression levels were calculated using the $2^{\Delta \mathrm{Ct}}$ method. Standard deviations were calculated from technical triplicate reactions and were represented as error bars. Primers used can be found in Supplementary Data 1. 
ChIP-qPCR signals were calculated as $\%$ of input using technical triplicates. Each ChIP sample was normalized to the average signals obtained in the same sample when using negative control regions primers (Chr2_neg and Chr6_neg; see Supplementary Data 1). Standard deviations were calculated from technical triplicate reactions and represented as error bars.

\section{C-seq analysis}

All 4C-seq samples were sequenced on an Illumina HiSeq 2500 sequencer, generating single reads of 74 bases in length. From these reads, the sequence attached to the viewpoint was extracted starting before the restriction site for NlaIII (CATG). These sequences were trimmed down to 41 base-pairs and aligned to the mouse GRCm38/mm10 reference genome using the HISAT2 aligner ${ }^{87}$. From these alignments, RPM (reads per million) normalized bedgraph files were generated for downstream visualization and analysis ${ }^{88}$.

\section{Gene Annotation}

The RefSeq gene annotation was downloaded through the UCSC Table Browser (https://genome.ucsc.edu/cgi-bin/hgTables) and used for the different analyses,

\section{ChIP-Seq and PRO-Seq pre-processing steps}

For all ChIP-Seq or PRO-Seq samples analyzed from fastq reads, the read quality was assessed with FastQC (http://www.bioinformatics.babraham.ac.uk/projects/fastqc/) and MultiQC ${ }^{95}$.

For ChIP-Seq data, the removal of read adapters and low quality filtering was done with trimmomatic ${ }^{96}$.

For PRO-Seq data, adapter removal was performed with cutadapt $1.18^{97}$ filtering for a minimum of 15 bases (adapter sequence: TGGAATTCTCGGGTGCCAAGG). In addition, reads mapping to the mouse rDNA repeats (GenBank: BK000964.3) were discarded from downstream analysis.

For both data types, reads were mapped to the mouse mm9 reference genome with Bowtie $2^{98}$. Last, for all ChIP-Seq samples, upon read mapping, duplicated reads were discarded, with the usage of SAMtools ${ }^{99}$.

\section{Genetic properties of CGIs}




\section{Data retrieval and pre-processing}

To perform the analyses described below, the required data sets were: PE coordinates, CGI coordinates and a list of developmental genes.

Poised enhancer coordinates were downloaded from ${ }^{9}$ and converted from $\mathrm{mm} 10$ to $\mathrm{mm} 9$ mouse genome coordinate with the UCSC LiftOver tool (https://genome.ucsc.edu/cgibin/hgLiftOver). Only poised enhancers more than $2.5 \mathrm{~kb}$ away from any TSS (PE-all) were considered. Among them, those PEs that were at least 10kb away from any TSS are referred to as $P E$-distal. All RefSeq transcripts were considered for this PE classification.

NMI coordinates were obtained from ${ }^{28}$. CAP-CGI coordinates were obtained from ${ }^{7}$, without applying any extension.

A relevant feature for many important cell-identity genes is that they are frequently embedded within broad domains of H3K27me3 when they are not expressed ${ }^{100,101}$. Therefore, we processed H3K27me3 ChIP-Seq and its corresponding input data generated in mESC (GSE89209; H3K27me3 ID: SRR4453259, Input ID: SRR4453262). Next, H3K27me3 peaks with respect to the Input were called with MACS2 ${ }^{102}$ using the broad peak calling mode. Only those peaks with a fold-enrichment $>3$ and q value $<0.1$ were maintained. Subsequently, peaks closer than $1 \mathrm{~kb}$ were merged using bedtools, and associated with a protein coding gene if they overlapped a TSS. Lastly, the size distribution of the H3K27me3 peaks associated with genes was studied and developmental genes were defined as those genes with the largest peak sizes. To do so, the knee of the size distribution was determined with findiplist() (inflection $\mathrm{R}$ package; [https://cran.r-project.org/web/packages/inflection/vignettes/inflection.html]). Upon curvature analysis, a threshold of $6 \mathrm{~Kb}$ was defined and, thus, all genes with a H3K27me3 peak length larger than 6kb were considered as developmental genes (devTSS).

\section{CGI groups classification}

NMI and CAP-CGI were associated with PE-distal or devTSS if located less than $3 \mathrm{~kb}$ away from them. In addition, to get a representation of the bulk genome composition, a third group of random regions was created (generated independently for NMI and CAP-CGI sequence composition analysis). To create this random group, the set of regions (NMI or CAP-CGI) in proximity to PE-distal was taken as reference in terms of region sizes. Then, each of the regions associated with a PE-distal was randomly relocated along the genome 1000 times (maintaining its size). Finally the set of all randomized regions constituted the random group. 


\section{Sequence Composition Calculations}

To retrieve the DNA sequences of the studied regions, the BSgenome package was used ${ }^{103}$, using as reference the unmasked mouse mm9 genome. For each region: its length, the percentage in $\mathrm{G}+\mathrm{C}$, the percentage in $\mathrm{CpG}$ and the $\mathrm{CpG}$ observed/expected ratio was calculated. The $\% \mathrm{CpG}$ was calculated as the ratio of $\mathrm{CpG}$ dinucleotide counts with respect to half the total region length. The ratio of observed to expected $\mathrm{CpG}$ is calculated according to the formula presented in ${ }^{104}$.

\section{Comparison of eRNA levels between different classes of active enhancers}

\section{Data retrieval and pre-processing}

The required data sets were: active enhancer coordinates, TAD maps, RNA-Seq, H3K27ac and PRO-Seq data from WT mESC. The active enhancer coordinates and the RNA-Seq data were used in both of the analyses described below.

Gene expression data (RNA-Seq FPKMs) and active enhancer coordinates from WT mESC were obtained from ${ }^{9}$. Enhancer coordinates were converted from mm10 to $\mathrm{mm} 9$ genome version with the UCSC LiftOver tool (https://genome.ucsc.edu/cgi-bin/hgLiftOver). To avoid confounding effects between transcripts produced by enhancers or genes, only active intergenic enhancers located at least $10 \mathrm{~kb}$ away from any TSS and 20kb from any transcription termination site (TTS) were considered.

Regarding the H3K27ac ChIP-Seq data from mESC, for the analyses presented in Fig 4 and Extended Fig 11a, the fastq raw data was retrieved from GEO (GSE89209; sample ID: SRR4453258) and pre-processed as indicated above. For the analyses presented in Extended Fig 11b-c, two H3K27ac bigWig files (from two replicates) were downloaded from GEO (samples IDs: GSM2808655 and GSM2808669).

With respect to the PRO-Seq data from $\mathrm{mESC}$, for the analyses presented in Fig 4 and Extended Fig 11a, the fastq raw data was obtained from GEO (GSE115713; sample IDs: SRR7300121, SRR7300122). The data from the two replicates was combined and preprocessed as described above. For the analyses presented in Extended Fig 11b-c, two PROSeq bigWig files (one for the forward DNA strand and the other for the reverse one) derived from the combination of three replicates, were obtained from GEO (GSE130691). 
TAD maps from mESC were retrieved from ${ }^{33}$. For the analyses presented in Fig 4 and Extended Fig 11a, the TAD map used was mESC_Dixon2012-raw_TADs.txt. For the analyses presented in Extended Fig 11b-c, the TAD map used was mESC.Bonev_2017raw.domains, whose coordinates were converted from mm10 to mm9 with UCSC LiftOver tool (https://genome.ucsc.edu/cgi-bin/hgLiftOver).

\section{H3K27ac \& PRO-Seq enhancer levels quantification}

To quantify H3K27ac and PRO-Seq enhancer levels two different approaches were followed, one for the analyses presented in Fig 4 and Extended Fig 11a .and another one for the analyses presented in Extended Fig 11b-c.

Fig 4 and Extended Fig 11a: H3K27ac and PRO-Seq reads presenting a mapping quality less than 10 were discarded using SAMtools ${ }^{99}$. Next, with the resulting mapped reads in BAM format, a bigwig was generated with deepTools ${ }^{105}$ and bamCoverage tool (RPGC normalization, and 1870000000 effective genome size). Finally, H3K27ac and PRO-Seq enhancer mean scores were obtained with the computeMatrix tool from deepTools, taking as input the active enhancer coordinates and the H3K27ac and PRO-Seq bigwigs. For H3K27ac, the signals were calculated $+/-1 \mathrm{~kb}$ with respect to the enhancer midpoints, while for PRO-Seq $\mathrm{a}+/-0.5 \mathrm{~kb}$ window was used instead.

Extended Fig 11b-c: H3K27ac and PRO-Seq mean signals for the active enhancers were calculated with the bigWigAverageOverBed UCSC binary tool. Next, PRO-Seq signals for each enhancer from the two different strands were averaged and the same was done for the signals coming from the different $\mathrm{H} 3 \mathrm{~K} 27 \mathrm{ac}$ replicates.

\section{Active enhancers classification}

Three groups of active enhancers were defined according to the following criteria. ( $I$ ) enhancers located in TADs containing only poorly expressed genes (all genes with $<0.5$ FPKM); (II) enhancers located in a TAD with at least one gene with expression levels $>10$ FPKM; (III) enhancers whose closest gene within the same TAD has expression levels $>10$ FPKM.

\section{Balancing of H3K27ac levels within enhancer classes}

To evaluate whether differences in eRNA levels between the three groups of active enhancers could be simply explained by differences in $\mathrm{H} 3 \mathrm{~K} 27 \mathrm{ac}$, the levels of this histone modification were balanced in some of the presented analyses (Extended Fig 11a,c). Briefly, enhancers 
with similar H3K27ac levels belonging to the three enhancer classes were selected by applying the nearest neighbour matching method (without replacement and ratio $=1$ ) using MatchIt [ https://cran.r-project.org/web/packages/MatchIt/MatchIt.pdf] and considering the enhancer group (I) as the treatment condition.

\section{Cliff's delta effect size estimator}

Cliff's delta ${ }^{106,107}$, a non-parametric effect size estimator, was used to quantify and interpret the differences between different groups of genomic regions for different types of genetic, transcriptional or epigenetic features. This measure relies on the concept of dominance (which refers to the degree of overlap between distributions) rather than means (as in conventional effect size indices such as Cohen's $d$ ) and is considered to be more robust when signal distributions are skewed ${ }^{108}$. Cliff's delta, was estimated using the cliff.delta() function from the R package effsize [https://cran.r-project.org/web/packages/effsize/index.html]. Differences between groups with an associated $\mid$ delta $\mid<0.147$ can be considered as negligible and $\mid$ delta $\mid>=$ 0.147 as non-negligible.

\section{References}

1. Deaton, A. M. \& Bird, A. CpG islands and the regulation of transcription. Genes Dev. 25, 1010-1022 (2011).

2. Guenther, M. G., Levine, S. S., Boyer, L. A., Jaenisch, R. \& Young, R. A. A Chromatin Landmark and Transcription Initiation at Most Promoters in Human Cells. Cell 130, 77-88 (2007).

3. Weber, M. et al. Distribution, silencing potential and evolutionary impact of promoter DNA methylation in the human genome. Nat. Genet. 39, 457-466 (2007).

4. Hartl, D. et al. CG dinucleotides enhance promoter activity independent of DNA methylation. Genome Res. 29, 554-563 (2019).

5. Bell, J. S. K. \& Vertino, P. M. Orphan $\mathrm{CpG}$ islands define a novel class of highly active enhancers. Epigenetics 12, 449-464 (2017).

6. Sarda, S., Das, A., Vinson, C. \& Hannenhalli, S. Distal CpG islands can serve as alternative promoters to transcribe genes with silenced proximal-promoters. Genome Res. 27, 553-566 (2017).

7. Illingworth, R. S. et al. Orphan CpG Islands Identify numerous conserved promoters in the mammalian genome. PLoS Genet. 6, e1001134 (2010).

8. Rada-Iglesias, A. et al. A unique chromatin signature uncovers early developmental enhancers in humans. Nature 470, 279-83 (2011).

9. Cruz-Molina, S. et al. PRC2 Facilitates the Regulatory Topology Required for Poised Enhancer Function during Pluripotent Stem Cell Differentiation. Cell Stem Cell 20, 1-17 (2017).

10. Furlong, E. E. M. \& Levine, M. Developmental enhancers and chromosome topology. Science 361, 1341-1345 (2018). 
11. Andrey, G. \& Mundlos, S. The three-dimensional genome : regulating gene expression during pluripotency and development. 144, 3646-3658 (2017).

12. Spielmann, M., Lupiáñez, D. G. \& Mundlos, S. Structural variation in the 3D genome. Nat. Rev. Genet. 19, 453-467 (2018).

13. de Laat, W. \& Duboule, D. Topology of mammalian developmental enhancers and their regulatory landscapes. Nature 502, 499-506 (2013).

14. Spitz, F. \& Furlong, E. E. M. Transcription factors: From enhancer binding to developmental control. Nat. Rev. Genet. 13, 613-626 (2012).

15. Long, H. K., Prescott, S. L. \& Wysocka, J. Ever-Changing Landscapes: Transcriptional Enhancers in Development and Evolution. Cell 167, 1170-1187 (2016).

16. Kvon, E. Z. Using transgenic reporter assays to functionally characterize enhancers in animals. Genomics 106, 185-192 (2015).

17. Dixon, J. R. et al. Topological domains in mammalian genomes identified by analysis of chromatin interactions. Nature 485, 376-380 (2012).

18. Dowen, J. M. et al. Control of cell identity genes occurs in insulated neighborhoods in mammalian chromosomes. Cell 159, 374-387 (2014).

19. Nora, E. P. et al. Spatial partitioning of the regulatory landscape of the X-inactivation centre. Nature 485, 381-385 (2012).

20. Laugsch, M. et al. Modeling the Pathological Long-Range Regulatory Effects of Human Structural Variation with Patient-Specific hiPSCs. Cell Stem Cell 24, 736-752.e12 (2019).

21. Rao, S. S. P. et al. Cohesin Loss Eliminates All Loop Domains. Cell 171, 305-320.e24 (2017).

22. Nora, P. et al. Targeted Degradation of CTCF Decouples Local Insulation of Chromosome Domains from Genomic Compartmentalization. Cell 169, 930-944 (2017).

23. Ghavi-Helm, Y. et al. Highly rearranged chromosomes reveal uncoupling between genome topology and gene expression. Nat. Genet. 51, 1272-1282 (2019).

24. Arnold, C. D. et al. Genome-wide assessment of sequence-intrinsic enhancer responsiveness at single-base-pair resolution. Nat. Biotechnol. 35, 136-144 (2016).

25. Haberle, V. et al. Transcriptional cofactors display specificity for distinct types of core promoters. Nature 570, 122-126 (2019).

26. Steinhaus, R., Gonzalez, T., Seelow, D. \& Robinson, P. N. Pervasive and CpG-dependent promoter-like characteristics of transcribed enhancers. Nucleic Acids Res. 48, 5306-5317 (2020).

27. Bogdanović, O. et al. Active DNA demethylation at enhancers during the vertebrate phylotypic period. Nat. Genet. 48, 417-426 (2016).

28. Long, H. K. et al. Epigenetic conservation at gene regulatory elements revealed by nonmethylated DNA profiling in seven vertebrates. Elife 2, 1-19 (2013).

29. Farley, E. K. et al. Suboptimization of developmental enhancers. Science 350, 325-328 (2015).

30. Bolt, C. C. \& Duboule, D. The regulatory landscapes of developmental genes. Development 147, 1-7 (2020).

31. Long, H. K., Blackledge, N. P. \& Klose, R. J. ZF-CxxC domain-containing proteins, CpG islands and the chromatin connection. Biochem. Soc. Trans. 41, 727-740 (2013).

32. Yao, X. et al. Tild-CRISPR Allows for Efficient and Precise Gene Knockin in Mouse and Human Cells. Dev. Cell 45, 526-536.e5 (2018). 
33. Wang, Y. et al. The 3D Genome Browser: A web-based browser for visualizing 3D genome organization and long-range chromatin interactions. Genome Biol. 19, 1-12 (2018).

34. Yan, J. et al. Histone H3 lysine 4 monomethylation modulates long-range chromatin interactions at enhancers. Cell Res. 28, 204-220 (2018).

35. Pope, B. D. et al. Topologically associating domains are stable units of replication-timing regulation. Nature 515, 402-405 (2014).

36. Wachter, E. et al. Synthetic CpG islands reveal DNA sequence determinants of chromatin structure. Elife 3, 1-16 (2014).

37. Diez, R. \& Storey, K. G. Markers in vertebrate neurogenesis. Nat. Rev. Neurosci. 2, 835-839 (2001).

38. Bentovim, L., Harden, T. T. \& DePace, A. H. Transcriptional precision and accuracy in development: From measurements to models and mechanisms. Dev. 144, 3855-3866 (2017).

39. Iguchi-Ariga, S. M. \& Schaffner, W. CpG methylation of the cAMP-responsive enhancer/promoter sequence TGACGTCA abolishes specific factor binding as well as transcriptional activation. Genes Dev. 3, 612-619 (1989).

40. Boyes, J. \& Bird, A. DNA methylation inhibits transcription indirectly via a methyl-CpG binding protein. Cell 64, 1123-1134 (1991).

41. Jones, P. L. et al. Methylated DNA and MeCP2 recruit histone deacetylase to repress transcription. Nat. Genet. 19, 187-191 (1998).

42. Klemm, S. L., Shipony, Z. \& Greenleaf, W. J. Chromatin accessibility and the regulatory epigenome. Nat. Rev. Genet. 20, 207-220 (2019).

43. Fenouil, R. et al. CpG islands and GC content dictate nucleosome depletion in a transcriptionindependent manner at mammalian promoters. Genome Res. 22, 2399-2408 (2012).

44. You, J. S. et al. OCT4 establishes and maintains nucleosome-depleted regions that provide additional layers of epigenetic regulation of its target genes. Proc. Natl. Acad. Sci. 108, 1449714502 (2011).

45. Stadler, M. B. et al. DNA-binding factors shape the mouse methylome at distal regulatory regions. Nature 480, 490-495 (2011).

46. Creyghton, M. P. et al. Histone H3K27ac separates active from poised enhancers and predicts developmental state. Proc. Natl. Acad. Sci. 107, 21931-21936 (2010).

47. Visel, A. et al. ChIP-seq accurately predicts tissue-specific activity of enhancers. Nature 457, 854-858 (2009).

48. Bird, A. P. DNA methylation and the frequency of $\mathrm{CpG}$ in animal DNA. Nucleic Acids Res. 8, 1499-1504 (1980).

49. Kim, T.-K. et al. Widespread transcription at neuronal activity-regulated enhancers. Nature 465, 182-187 (2010).

50. Hirabayashi, S. et al. NET-CAGE characterizes the dynamics and topology of human transcribed cis-regulatory elements. Nat. Genet. 51, 1369-1379 (2019).

51. Fitz, J. et al. Spt5-mediated enhancer transcription directly couples enhancer activation with physical promoter interaction Johanna. Nat. Genet. 52, 505-515 (2020).

52. Bell, E. et al. Dynamic CpG methylation delineates subregions within super-enhancers selectively decommissioned at the exit from naive pluripotency. Nat. Commun. 11, 1-16 (2020). 
53. El Khattabi, L. et al. A Pliable Mediator Acts as a Functional Rather Than an Architectural Bridge between Promoters and Enhancers. Cell 178, 1145-1158 (2019).

54. Denholtz, M. et al. Long-Range Chromatin Contacts in Embryonic Stem Cells Reveal a Role for Pluripotency Factors and Polycomb Proteins in Genome Organization. Cell Stem Cell 13, 602-616 (2013).

55. Wang, J. et al. A protein interaction network for pluripotency of embryonic stem cells. Nature 444, 364-368 (2006).

56. Pachano, T., Crispatzu, G. \& Rada-iglesias, A. Polycomb proteins as organizers of 3D genome architecture in embryonic stem cells. Brief. Funct. Genomics 18, 358-366 (2019).

57. Bantignies, F. et al. Polycomb-dependent regulatory contacts between distant hox loci in drosophila. Cell 144, 214-226 (2011).

58. Mas, G. \& Di Croce, L. The role of Polycomb in stem cell genome architecture. Curr. Opin. Cell Biol. 43, 87-95 (2016).

59. Yan, J. et al. Histone H3 lysine 4 monomethylation modulates long-range chromatin interactions at enhancers. Cell Res. 28, 204-220 (2018).

60. Kundu, S. et al. Polycomb Repressive Complex 1 Generates Discrete Compacted Domains that Change during Differentiation. Mol. Cell 65, 432-446 (2017).

61. Isono, K. et al. SAM domain polymerization links subnuclear clustering of PRC1 to gene silencing. Dev. Cell 26, 565-577 (2013).

62. Boyle, S. et al. A central role for canonical PRC1 in shaping the 3D nuclear landscape. Genes Dev. 34, 931-949 (2020).

63. Loubiere, V., Papadopoulos, G. L., Szabo, Q., Martinez, A. M. \& Cavalli, G. Widespread activation of developmental gene expression characterized by PRC1-dependent chromatin looping. Sci. Adv. 6, eaax4001 (2020).

64. Andersson, R. \& Sandelin, A. Determinants of enhancer and promoter activities of regulatory elements. Nat. Rev. Genet. 21, 71-87 (2020).

65. Bonev, B. et al. Multiscale 3D Genome Rewiring during Mouse Neural Development. Cell 171, 557-572 (2017).

66. Lloret-Llinares, M. et al. The RNA exosome contributes to gene expression regulation during stem cell differentiation. Nucleic Acids Res. 46, 11502-11513 (2018).

67. Andersson, R., Sandelin, A. \& Danko, C. G. A unified architecture of transcriptional regulatory elements. Trends Genet. 31, 426-433 (2015).

68. Kim, T. K. \& Shiekhattar, R. Architectural and Functional Commonalities between Enhancers and Promoters. Cell 162, 948-959 (2015).

69. Local, A. et al. Identification of $\mathrm{H} 3 \mathrm{~K} 4 \mathrm{me} 1$-associated proteins at mammalian enhancers. Nat. Genet. 50, 73-82 (2018).

70. Schoenfelder, S. et al. Polycomb repressive complex PRC1 spatially constrains the mouse embryonic stem cell genome. Nat. Genet. 47, 1179-86 (2015).

71. Gómez-Marín, C. et al. Evolutionary comparison reveals that diverging CTCF sites are signatures of ancestral topological associating domains borders. Proc Natl Acad Sci U S A 112, 7542-7547 (2015).

72. O'Brien, L. L. et al. Transcriptional regulatory control of mammalian nephron progenitors revealed by multi-factor cistromic analysis and genetic studies. PLoS Genetics vol. 14 (2018). 
73. van Arensbergen, J., van Steensel, B. \& Bussemaker, H. J. In search of the determinants of enhancer-promoter interaction specificity. Trends Cell Biol. 24, 695-702 (2014).

74. Catarino, R. R. \& Stark, A. Assessing sufficiency and necessity of enhancer activities for gene expression and the mechanisms of transcription activation. Genes Dev. 32, 202-223 (2018).

75. Lupiáñez, D. G. et al. Disruptions of topological chromatin domains cause pathogenic rewiring of gene-enhancer interactions. Cell 161, 1012-1025 (2015).

76. Guo, Y. et al. CRISPR Inversion of CTCF Sites Alters Genome Topology and Enhancer/Promoter Function. Cell 162, 900-910 (2015).

77. Despang, A. et al. Functional dissection of the Sox9-Kcnj2 locus identifies nonessential and instructive roles of TAD architecture. Nat. Genet. 51, 1263-1271 (2019).

78. Kragesteen, B. K. et al. Dynamic 3D chromatin architecture contributes to enhancer specificity and limb morphogenesis ,. Nat. Genet. 50, 1463-1473 (2018).

79. Li, X. \& Noll, M. Compatibility between enhancers and promoters determines the transcriptional specificity of gooseberry and gooseberry neuro in the Drosophila embryo. EMBO J. 13, 400-406 (1994).

80. Merli, C., Bergstrom, D. E., Cygan, J. A. \& Blackman, R. K. Promoter specificity mediates the indepeident regulation of neighboring genes. Genes Dev. 10, 1260-1270 (1996).

81. Zabidi, M. A. et al. Enhancer-core-promoter specificity separates developmental and housekeeping gene regulation. Nature 518, 556-559 (2015).

82. Perino, M. et al. MTF2 recruits Polycomb Repressive Complex 2 by helical-shape-selective DNA binding. Nat. Genet. 50, 1002-1010 (2018).

83. Blackledge, N. P. et al. Variant PRC1 complex-dependent H2A ubiquitylation drives PRC2 recruitment and polycomb domain formation. Cell 157, 1445-1459 (2014).

84. Plys, A. J. et al. Phase separation and nucleosome compaction are governed by the same domain of Polycomb Repressive Complex 1. bioRxiv (2018) doi:https://doi.org/10.1101/467316.

85. Beltran, M. et al. The interaction of PRC2 with RNA or chromatin s mutually antagonistic. Genome Res. 26, 896-907 (2016).

86. Sabari, B. R. et al. Coactivator condensation at super-enhancers links phase separation and gene control. Science 361, eaar3958 (2018).

87. Cho, W. K. et al. Mediator and RNA polymerase II clusters associate in transcriptiondependent condensates. Science 361, 412-415 (2018).

88. Shrinivas, K. et al. Enhancer Features that Drive Formation of Transcriptional Condensates. Mol. Cell 75, 549-561.e7 (2019).

89. Gouti, M. et al. In vitro generation of neuromesodermal progenitors reveals distinct roles for wnt signalling in the specification of spinal cord and paraxial mesoderm identity. PLoS Biol. 12, e1001937 (2014).

90. Matsuda, K. \& Kondoh, H. Dkk1-dependent inhibition of Wnt signaling activates Hesx1 expression through its 5??? enhancer and directs forebrain precursor development. Genes to Cells 19, 374-385 (2014).

91. Stadhouders, R. et al. Multiplexed chromosome conformation capture sequencing for rapid genome-scale high-resolution detection of long-range chromatin interactions. Nat. Protoc. 8 , 509-524 (2013).

92. Giresi, P. G., Kim, J., McDaniell, R. M., Iyer, V. R. \& Lieb, J. D. FAIRE (Formaldehyde- 
Assisted Isolation of Regulatory Elements) isolates active regulatory elements from human chromatin. Genome Res. 17, 877-85 (2007).

93. Taberlay, P. C., Statham, A. L., Kelly, T. K., Clark, S. J. \& Jones, P. A. Reconfiguration of nucleosome-depleted regions at distal regulatory elements accompanies DNA methylation of enhancers and insulators in cancer. Genome Res. 24, 1421-1432 (2014).

94. Requena, F. et al. NOMePlot: analysis of DNA methylation and nucleosome occupancy at the single molecule. Sci. Rep. 9, 1-10 (2019).

95. Ewels, P., Magnusson, M., Lundin, S. \& Käller, M. MultiQC: Summarize analysis results for multiple tools and samples in a single report. Bioinformatics 32, 3047-3048 (2016).

96. Bolger, A. M., Lohse, M. \& Usadel, B. Trimmomatic: A flexible trimmer for Illumina sequence data. Bioinformatics 30, 2114-2120 (2014).

97. Martin, M. Cutadapt removes adapter sequences from high-throughput sequencing reads. EMBnet.journal 17, 10-12 (2011).

98. Langmead, B. \& Salzberg, S. L. Fast gapped-read alignment with Bowtie 2. Nat. Methods 9, 357-359 (2012).

99. Li, H. et al. The Sequence Alignment/Map format and SAMtools. Bioinformatics 25, 20782079 (2009).

100. Rehimi, R. et al. Epigenomics-Based Identification of Major Cell Identity Regulators within Heterogeneous Cell Populations. Cell Rep. 17, 3062-3076 (2016).

101. Nakamura, R. et al. Large hypomethylated domains serve as strong repressive machinery for key developmental genes in vertebrates. Development 141, 2568-2580 (2014).

102. Feng, J., Liu, T., Qin, B., Zhang, Y. \& Liu, X. S. Identifying ChIP-seq enrichment using MACS. Nat. Protoc. 7, 1728-1740 (2012).

103. Pagès, H. BSgenome: Software infrastructure for efficient representation of full genomes and their SNPs. R package version 1.56.0. (2020).

104. Gardiner-Garden, M. \& Frommer, M. CpG islands in vertebrate genomes. J. Mol. Biol. 196, 261-282 (1987).

105. Ramírez, F. et al. deepTools2: a next generation web server for deep-sequencing data analysis. Nucleic Acids Res. 44, W160-W165 (2016).

106. Cliff, N. Dominance Statistics: Ordinal Analyses to Answer Ordinal Questions. Psychol. Bull. 114, 494-509 (1993).

107. Macbeth, G., Razumiejczyk, E. \& Ledesma, R. D. Cliff's Delta Calculator: A non-parametric effect size program for two groups of observations. Univ. Psychol. 10, 545-555 (2011).

108. Bush, S. J., McCulloch, M. E. B., Summers, K. M., Hume, D. A. \& Clark, E. L. Integration of quantitated expression estimates from polyA-selected and rRNA-depleted RNA-seq libraries. BMC Bioinformatics 18, 1-12 (2017).

109. Etchegaray, J. P. et al. The Histone Deacetylase SIRT6 Restrains Transcription Elongation via Promoter-Proximal Pausing. Mol. Cell 75, 683-699 (2019). 
Extended Data Fig. 1| Genetic features of the orphan CGIs associated with PEs. a, Percentage of poised enhancers (PEs) found within the indicated maximum distances $(0.25 \mathrm{~Kb}$ or $3 \mathrm{~Kb}$ ) to a computationally-defined CGI according to the following criteria: GC content > $50 \%$; Length > $200 \mathrm{bp}$; CpG (left panel) or a CAP-CGI identified with the CAP assay ${ }^{7}$ (right panel). b, Comparison of the $\mathrm{CpG}$ percentage, observed/expected $\mathrm{CpG}$ ratio, GC percentage and sequence length between random regions (see Methods), CAP-CGIs associated to poised enhancers (PE-CAP-CGI; blue) and CAP-CGIs associated to the TSS of developmental genes (devTSS-CAP-CGI; yellow; see Methods). On top of each plot, the asterisks indicate P-values calculated using unpaired Wilcoxon tests with Bonferroni correction for multiple testing $(* *=$ p.val $<1 \mathrm{e}^{-10}$; p.val $<0.05$ ); the numbers in black indicate the median fold changes between the indicated groups; the green numbers indicate non-negligible Cliff Delta effect sizes. 


\section{bioRxiv preprint doi: https://doi.org/10.11
(which was not certified by peer review)}

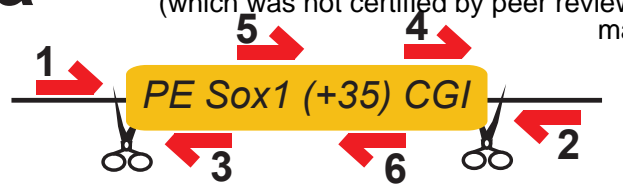

$1+2=1692(\mathrm{WT})$ or $552 \mathrm{bp}\left(\mathrm{PE}\right.$ Sox1 $\left.\mathrm{CGl}^{-{ }^{-}}\right)$

$1+3=255 \mathrm{bp} \quad 2+4=230 \mathrm{bp}$

$5+6=268 \mathrm{bp}$

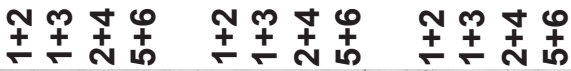

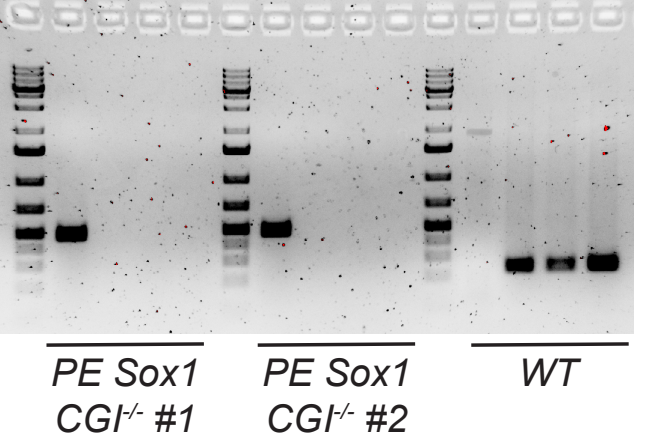

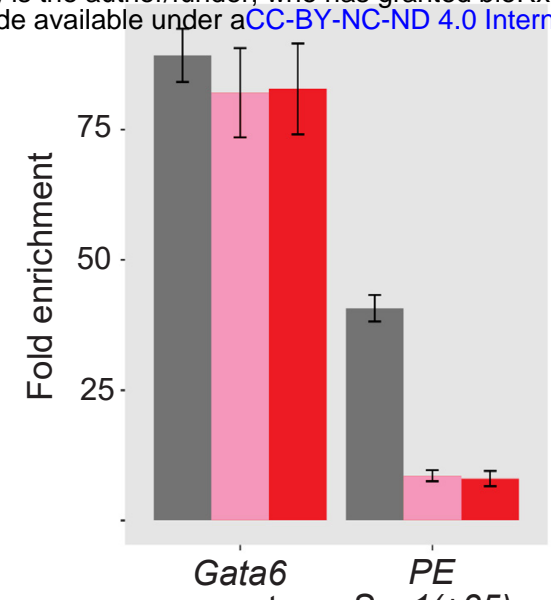

promoter Sox1(+35)

\section{WT}

PE Sox1 CG/ \#1

PE Sox1 CGI ${ }^{-/ 2}$

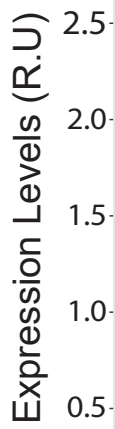

ESC

AntNPC
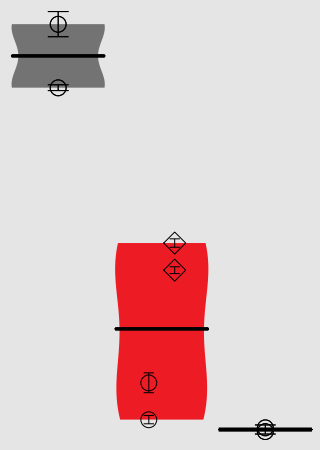

WT PE PE WT PE PE Sox1 Sox $1^{-1-}$ Sox1 Sox $1^{1-1}$ $\mathrm{CGI}^{-1-}$ $\mathrm{CGI}^{-1-}$

WT

$\begin{array}{ll}\text { PE Sox1 CGI- } & \bigcirc \text { Clone\#1 } \\ \text { PE Sox } 1^{-} & \diamond \text { Clone\#2 }\end{array}$ 
Extended Data Fig. 2| PE Sox1(+35)CGI recruits PcG and contributes to the cisregulatory function of $\boldsymbol{P E} \operatorname{Soxl}(+35)$. a, For the identification of the PE Soxl(+35)CGI deletion, primer pairs flanking each of the deletion breakpoints $(1+3$ and $4+2)$, located within the deleted region $(5+6)$ or amplifying a large or small fragment depending on the absence or presence of the deletion $(1+2)$ were used. The PCR results obtained for WT ESC and for two mESC clonal lines with homozygous deletions of the PE Soxl $(+35) C G I\left(P E \operatorname{Soxl}(+35) C^{-}-\right)$ are shown. b, H3K27me3 levels at PE Soxl(+35) were measured by ChIP-qPCR in WT mESC (grey), and in two PE Soxl $(+35) C G^{I-/}$ mESCs clones using primers adjacent to the deleted region. ChIP-qPCR signals were normalized against two negative regions (Supplementary Data 1). Error bars correspond to standard deviations from technical triplicates. c, Independent biological replicate for the data presented in Fig. 1d. The expression of Soxl was investigated by RT-qPCR in mESCs (left panel) and AntNPC (right panel) that were either WT (grey), homozygous for a deletion of the PE Soxl(+35) CGI (PE Soxl $\mathrm{CGI}^{--}$; red) or homozygous for the complete PE Soxl(+35) deletion ${ }^{9}$ (PE SoxI ${ }^{-/}$; black). Two different PE Soxl CGI ${ }^{-/}$mESC clones (circles and diamonds) and one PE SoxI ${ }^{-/}$clone were studied. For each cell line, two technical replicates of the AntNPC differentiation were performed. The plotted expression values of each clone correspond to the average and standard deviation (error bars) from three RT-qPCR technical replicates. Expression values were normalized to two housekeeping genes (Eefla and Hprt). 

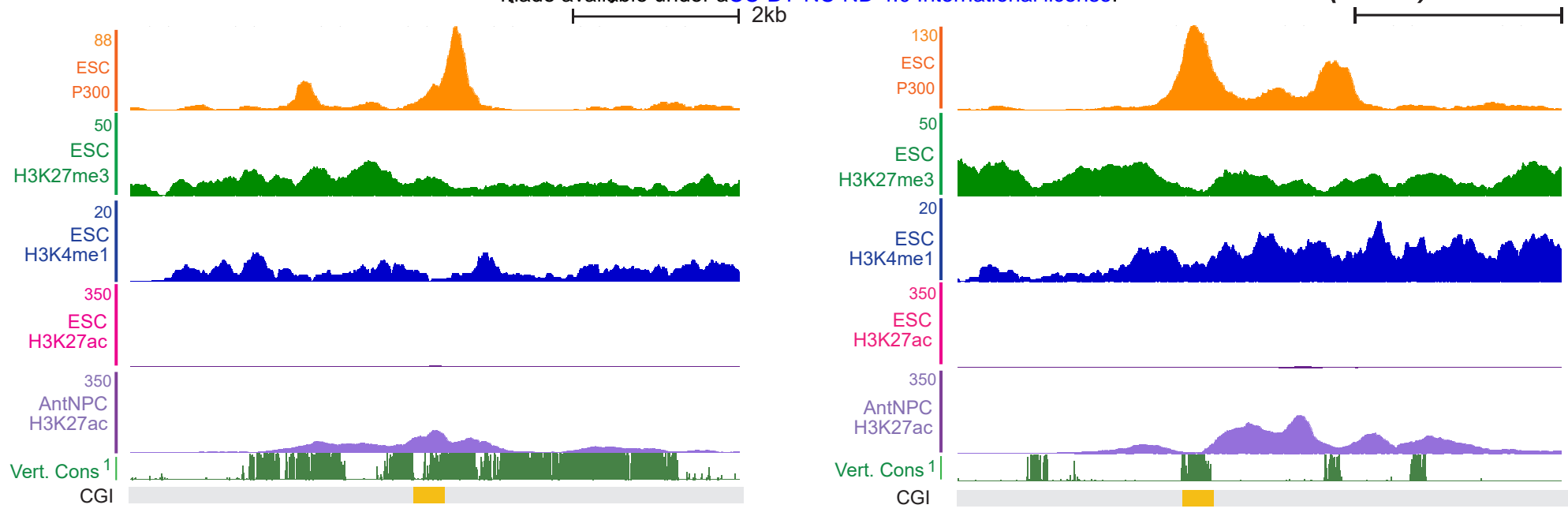

b

1+2 = 1428bp (TFBS), $1858 \mathrm{bp}(\mathrm{CGI})$,

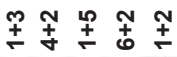

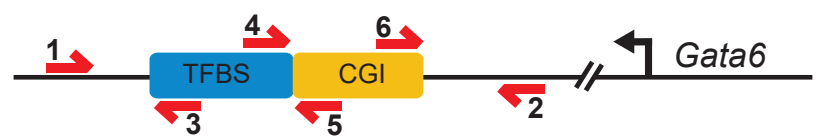

$2328 \mathrm{bp}($ TFBS $+\mathrm{CGI})$ or $901 \mathrm{bp}(\mathrm{WT})$

$\mathbf{1 + 3}=460 \mathrm{bp}$
$\mathbf{4 + 2}=716 \mathrm{bp}(\mathrm{TFBS})$ or $1616 \mathrm{bp}(\mathrm{TFBS}+\mathrm{CGI})$
$1+5$

$\mathbf{1 + 5}=649 \mathrm{bp}(\mathrm{CGI})$ or 1119bp $(\mathrm{TFBS}+\mathrm{CGI})$

6+2 $=708 \mathrm{bp}$

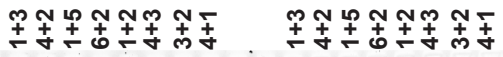

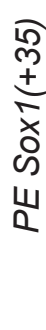

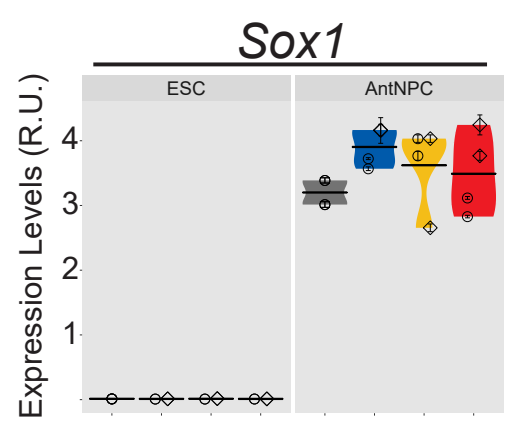

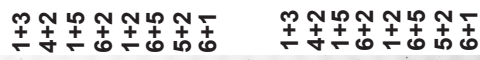
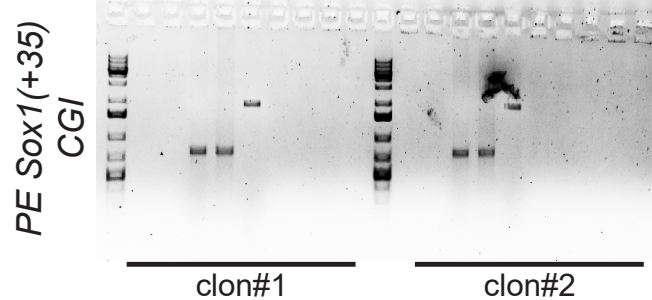

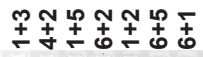
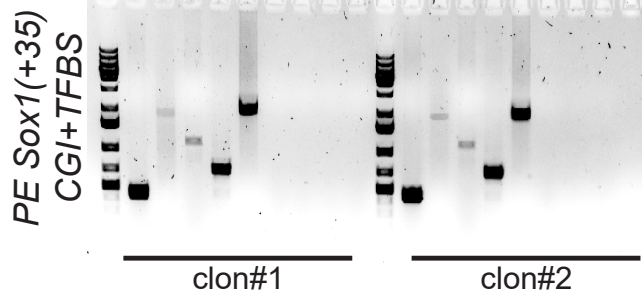

OClone\#1 $\diamond$ Clone\#2

WT TFBS CGI TFBS+CGI

\section{Gata6}

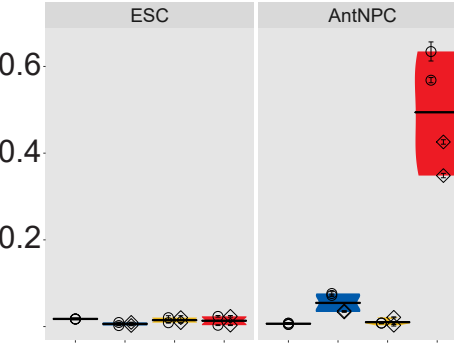


Extended Data Fig.3| Modular engineering of the PE Sox1(+35) within the Gata6-TAD.

a, Genome-browser view of the epigenomic and genomic features of two previously characterized PEs ${ }^{9}$ (left: PE Six3-(133); Right: PE Lmxlb(+59)) in which the oCGIs overlap with conserved sequences bound by P300 and, thus, likely to represent TFBS. The represented CGIs correspond to those computationally defined in the UCSC browser according to the following criteria: GC content > 50\%; Length > $200 \mathrm{bp}$; CpG Observed to expected ratio $>$ 0.6. Vert. Cons.= vertebrate PhastCons. b, For the identification of the different $P E$ Soxl $(+35)$ module insertions, primer pairs flanking the insertion borders $(1+3$ and $4+2 ; 1+5$ and $6+2$; or $1+3$ and $6+2)$, amplifying potential duplications $(4+3,3+2$ and $4+1$; or $6+5,5+2$ and $6+1$ ) and amplifying a large or small fragment depending on the absence or presence of the insertion $(1+2)$, respectively, were used. The PCR results obtained for WT $\mathrm{mESC}$ and for two mESC clonal lines with homozygous insertions for each of the three different combinations of $P E \operatorname{Soxl}(+35)$ modules (i.e. (i) $P E \operatorname{Soxl}(+35) T F B S$; (ii) $P E$ Soxl(+35)CGI; (iii) PE Soxl(+35)TFBS+CGI) inserted in the Gata6-TAD are shown. c, Independent biological replicate for the data presented in Fig. 2b. The expression of Gata6 and Soxl was measured by RT-qPCR in mESCs (left panels) and AntNPC (right panels) that were either WT (grey) or homozygous for the insertions of the different PE Soxl $(+35)$ modules (i.e. TFBS (blue), CGI (yellow), TFBS+CGI (red)). For the cells with the PE module insertions, two different clonal cell lines (circles and diamonds) were studied in each case. For each cell line, two technical replicates of the AntNPC differentiation were performed. The plotted expression values for each clone correspond to the average and standard deviation (error bars) from three RT-qPCR technical replicates. Expression values were normalized to two housekeeping genes (Eefla and Hprt). 


\section{Extended Data Fig.4| Modular engineering of the PE Sox1(+35) within the FoxA2-TAD} and of the $P E W n t 8 b(+21)$ within the Gata6-TAD. a, Strategy used to insert the $P E$ Soxl $(+35)$ components into the Foxa2-TAD. The upper left panel shows a close-up view of the epigenomic and genetic features of the PE Soxl $(+35)$ (Vert. Cons.= vertebrate PhastCons). The represented CGIs correspond to those computationally defined in the UCSC browser according to the following criteria: GC content > 50\%; Length > $200 \mathrm{bp}$; CpG Observed to expected ratio $>0.6$. The lower left panel shows the three combinations of $P E$ Soxl(+35) modules (i.e. (i) PE Soxl(+35)TFBS; (ii) $P E$ Soxl(+35)CGI; (iii) $P E$ Soxl $(+35) T F B S+C G I)$ inserted into the FoxA2-TAD. The right panel shows the TAD in which Foxa2 is included (i.e. Foxa2-TAD) according to publically available Hi-C data ${ }^{33,65}$; TAD boundaries are denoted with dotted lines; H3K27me3 ChIP-seq signals in mESC are shown in green"; CGIs are indicated as green rectangles; the red triangle indicates the integration site of the PE Soxl(+35) modules, approximately $100 \mathrm{~Kb}$ downstream of Foxa2. b, Strategy used to insert the $P E W n t 8 b(+21)$ components into the Gata6-TAD as described in (a). c-d, For identifying the successful insertion of the different $P E \operatorname{Sox} 1(+35)$ (c) or $P E$ $W n t 8 b(+21)(\mathrm{d})$ modules, primer pairs flanking the insertion borders $(1+3$ and $4+2 ; 1+5$ and $6+2$; or $1+3$ and $6+2)$, amplifying potential duplications $(4+3,3+2$ and $4+1$; or $6+5,5+2$ and $6+1)$ and amplifying a large or small fragment depending on the absence or presence of the insertion $(1+2)$, respectively, were used. The PCR results obtained for two mESC clonal lines with homozygous insertions for each of the three different combinations of PE Soxl $(+35)$ modules (i.e. (i) PE Soxl(+35)TFBS; (ii) PE Soxl(+35)CGI; (iii) PE Soxl(+35)TFBS+CGI) or $P E W n t 8 b(+21)$ modules (i.e. (i) $P E W n t 8 b(+21) T F B S$; (ii) $P E W n t 8 b(+21) C G I$; (iii) $P E$ Wnt $8 b(+21) T F B S+C G I)$ in the Foxa2-TAD (c) or Gata6-TAD (d), respectively, are shown. e-f, Independent biological replicate for the data shown in Fig. 2c (e) and Fig. 2d (f). The expression of Foxa2 (e), Gata6 (f), Soxl (e) and Wnt8b (f) was measured by RT-qPCR in mESCs (left panels) and AntNPC (right panels) that were either WT (grey) or homozygous for the insertions of the different PE Soxl(+35) (e) or Wnt8b(+21) (e) modules (i.e. TFBS (blue), CGI (yellow), TFBS+CGI (red)). For the cells with the PE module insertions, two different clonal cell lines (circles and diamonds) were studied in each case. For each cell line, two technical replicates of the AntNPC differentiation were performed. The plotted expression values for each clone correspond to the average and standard deviation (error bars) from three RT-qPCR technical replicates. Expression values were normalized to two housekeeping genes (Eefla and Hprt). 


\section{Extended Data Fig.5| Engineering of a PE Sox1(+35) construct with the TFBS module}

and an artificial CGI. a, Strategy used to insert the PE Sox $1(+35) T F B S$ alone of together with an artificial CGI (aCGI; see Methods) into the Gata6-TAD. The upper left panel shows a close-up view of the epigenomic and genetic features of the PE Sox1(+35) (Vert. Cons.= vertebrate PhastCons). The represented CGIs correspond to those computationally defined in the UCSC browser according to the following criteria: GC content > 50\%; Length > $200 \mathrm{bp}$; CpG Observed to expected ratio > 0.6. The lower left panel shows the two combinations of $P E \operatorname{Sox} 1(+35)$ modules (i.e. (i) PE Soxl(+35)TFBS; (ii) PE Soxl(+35)TFBS+aCGI) inserted into the Gata6 TAD. The right panel shows the TAD in which Gata6 is included (i.e. Gata6TAD) according to publically available $\mathrm{Hi}-\mathrm{C}$ data ${ }^{33,34}$; TAD boundaries are denoted with dotted lines; H3K27me3 ChIP-seq signals in mESC are shown in green ${ }^{9}$; CGIs are indicated as green rectangles; CTCF binding sites $^{35}$ are indicated as black rectangles; the red triangle indicates the integration site of the $P E \operatorname{Soxl}(+35)$ modules, approximately $100 \mathrm{~Kb}$ downstream of Gata6. b, For the identification of the PE Soxl(+35)TFBS+aCGI insertion, primer pairs flanking the insertion borders $(1+3$ and $4+2)$, amplifying potential duplications (4+3 and 4+4) and amplifying a large or small fragment depending on the absence or presence of the insertion $(1+2)$, respectively, were used. The PCR results obtained for two mESC clonal lines with homozygous insertions of PE Soxl(+35)TFBS $+a C G I$ in the Gata6-TAD are shown. c, Independent biological replicate for the data presented in Fig. 2e. The expression of Gata6 and Soxl was measured by RT-qPCR in mESCs (left panels) and AntNPC (right panels) that were either WT (grey) or homozygous for the PE Soxl(+35)TFBS (blue) or PE Soxl(+35)TFBS+aCGI (red) insertions. For the cells with the PE insertions, two different clonal cell lines (circles and diamonds) were studied in each case. For each cell line, two technical replicates of the AntNPC differentiation were performed. The plotted expression values for each clone correspond to the average and standard deviation (error bars) from three RT-qPCR technical replicates. Expression values were normalized to two house- keeping genes (Eefla and Hprt). 

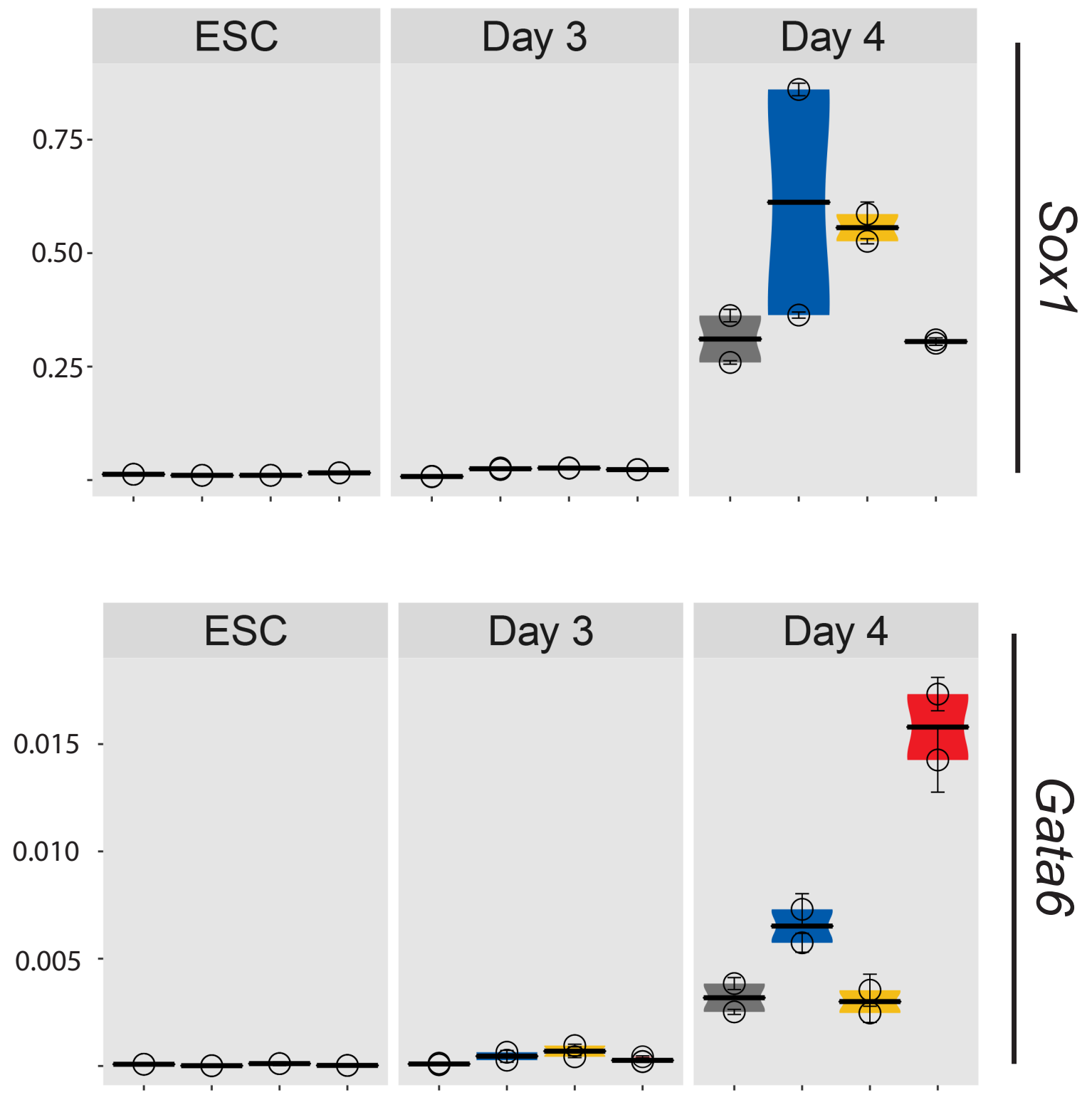

OClone\#1 WT WFBS CGI $\quad$ TFBS+CGI 
Extended Data Fig.6 Gata6 expression dynamics in cell lines with the PE Sox1(+35) modules inserted within the Gata6-TAD . The expression of Gata6 and Soxl was measured by RT-qPCR in mESCs and at intermediate stages of mESCs differentiation into AntNPC (Day 3 and Day 4). The analysed cells were either WT (grey) or homozygous for the insertions of the different PE Soxl(+35) modules (i.e. TFBS (blue), CGI (yellow), TFBS+CGI (red)) within the Gata6-TAD. For the cells with the PE module insertions, one clonal cell line (circles) was studied. For each cell line, two technical replicates of the AntNPC differentiation were performed. The plotted expression values for each clone correspond to the average and standard deviation (error bars) from three RT-qPCR technical replicates. Expression values were normalized to two housekeeping genes (Eefla and Hprt). 
bioRxiv preprint doi: https://doi.org/10.1101/2020.08.05.237768; this version pested August 5.2020. The copyright holder for this preprint a nof made available under aCC-BY-NC-ND 4.0 International license.
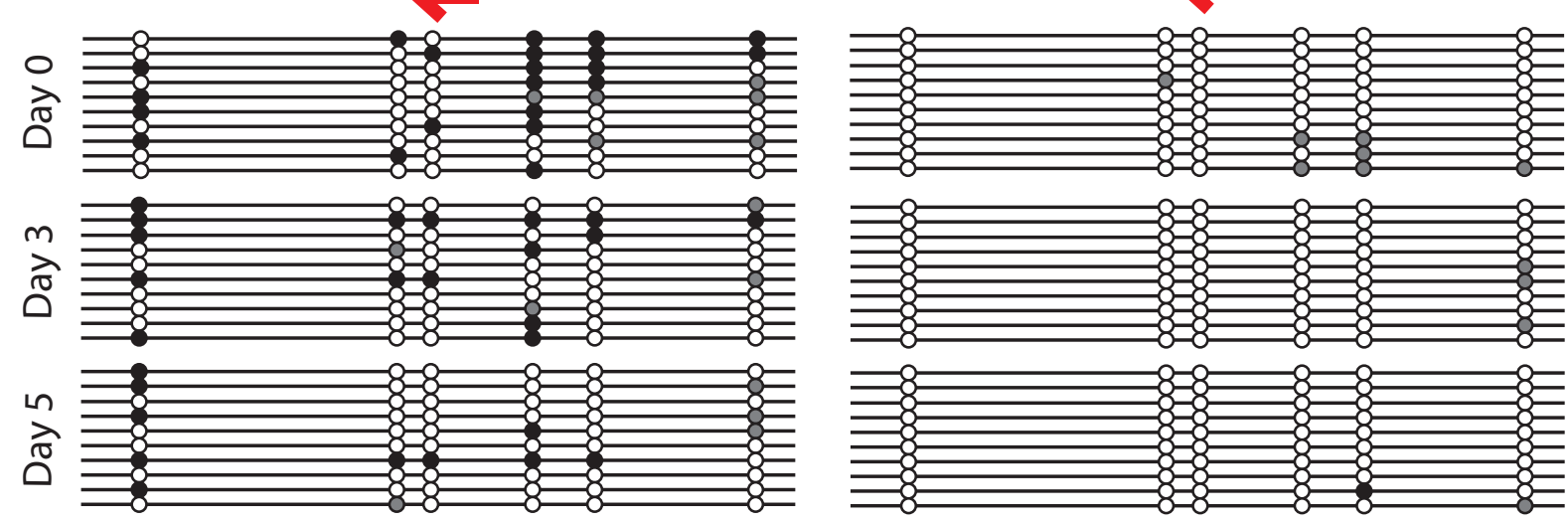

b

FAIRE
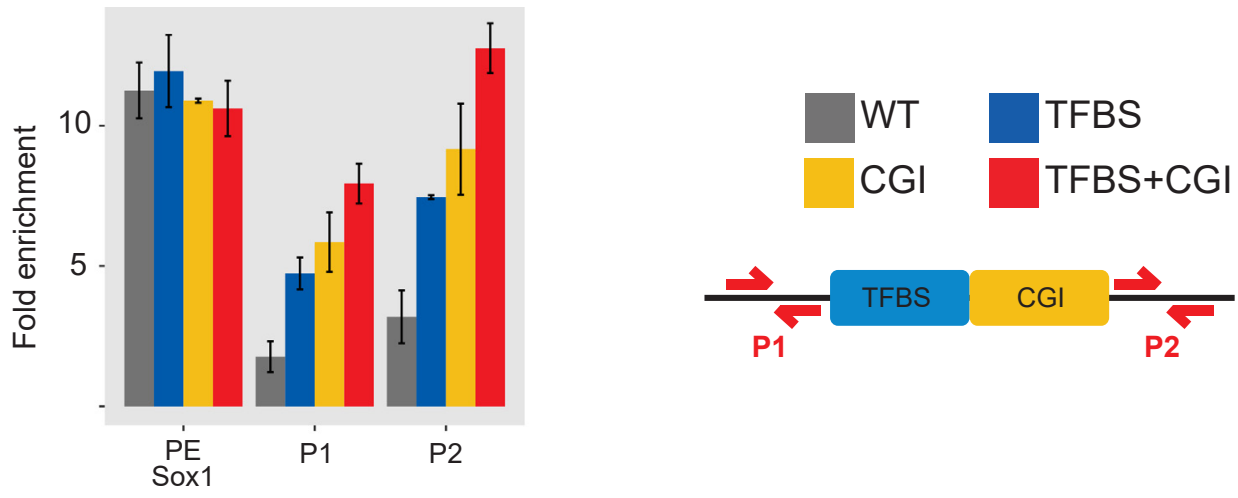

C
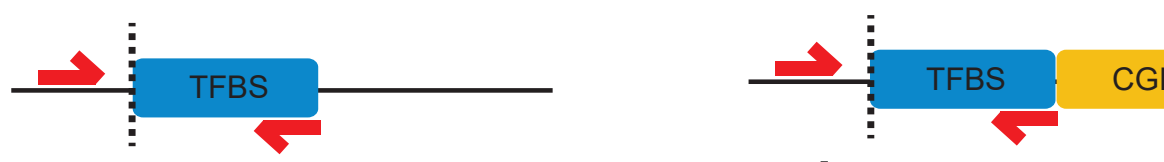

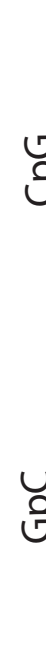
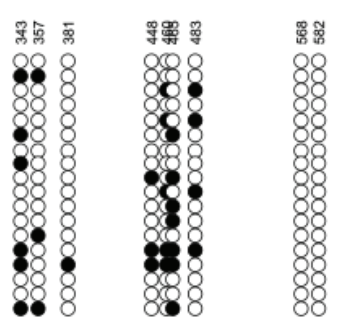

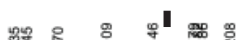

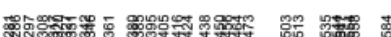

$\infty 0 \quad 0 \quad 0 \%$

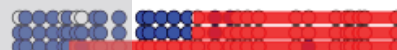
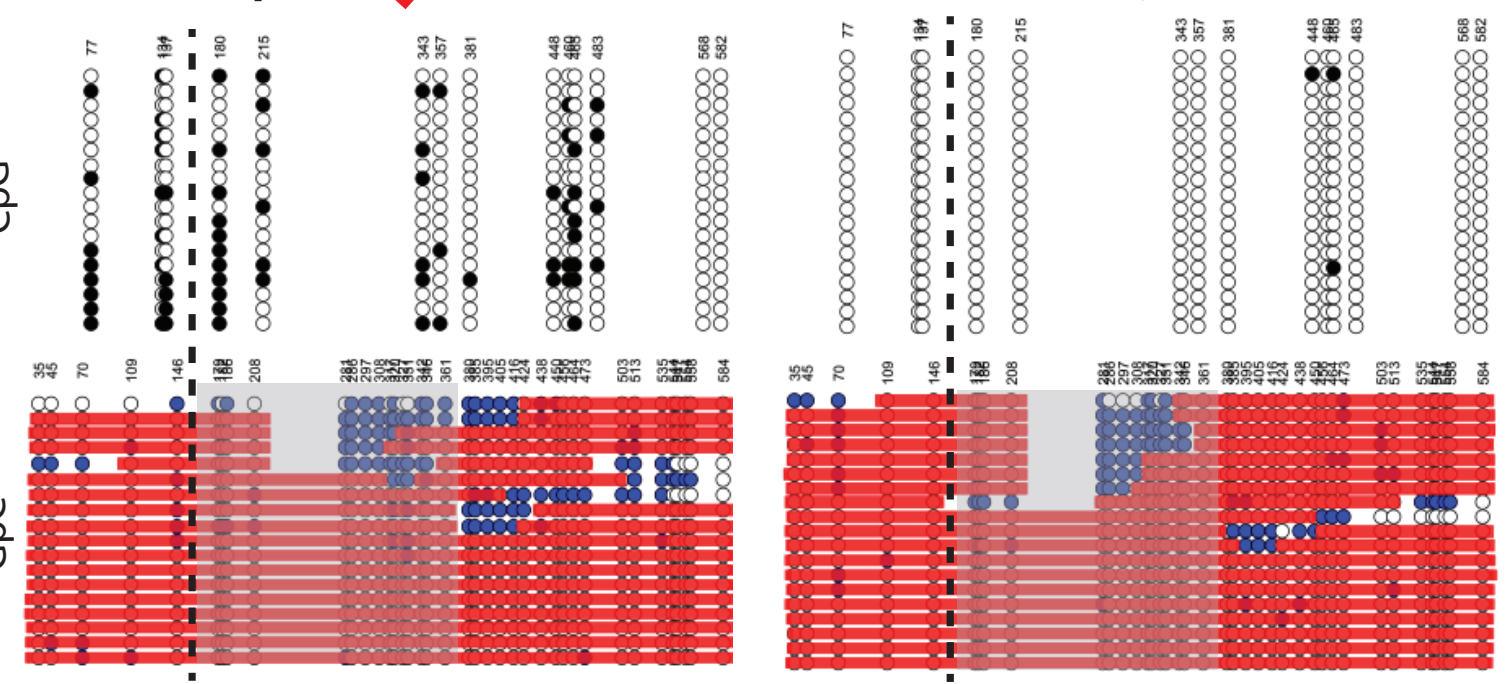

PE Sox1(+35)TFBS
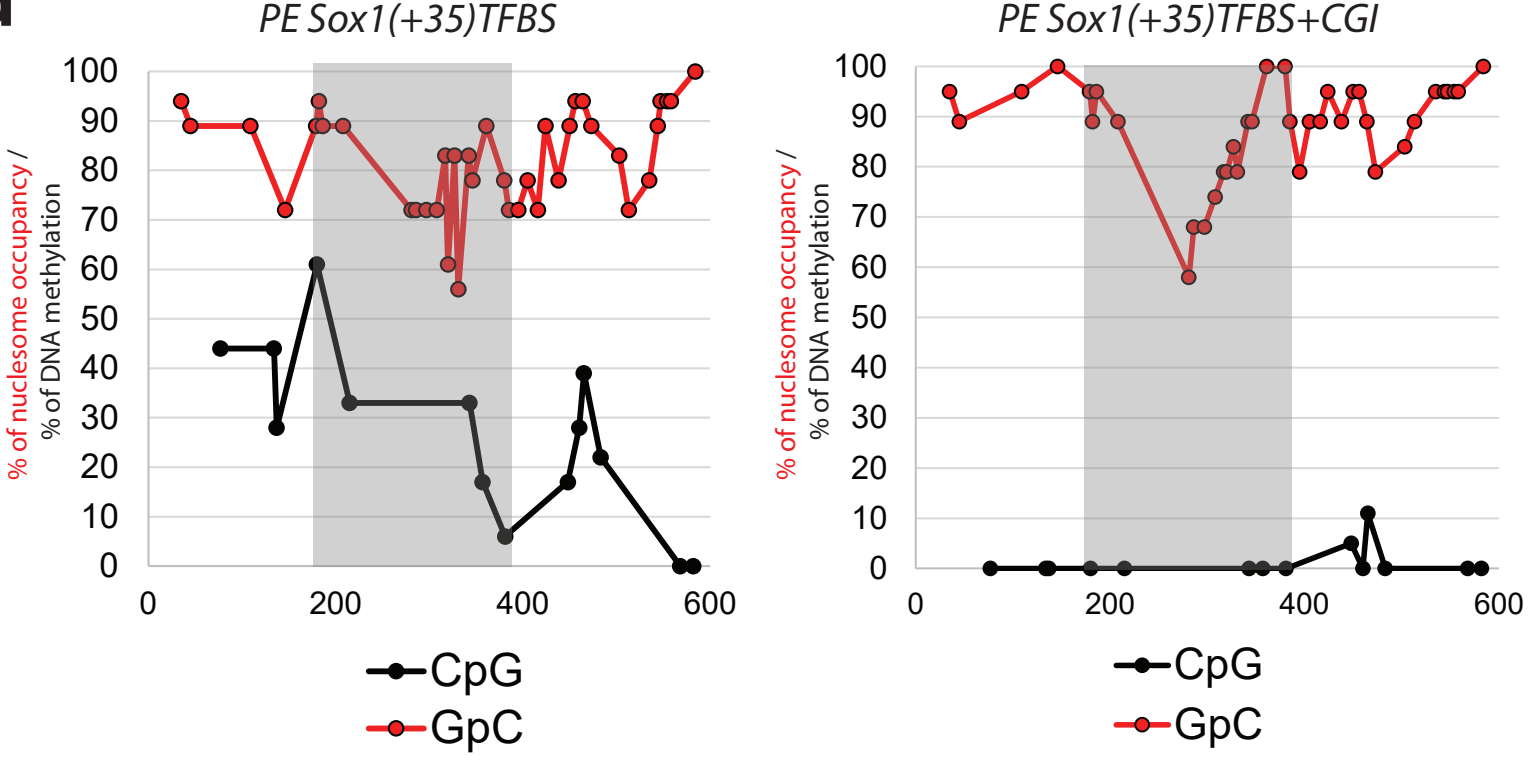


\section{Extended Data Fig.7| Epigenetic characterization of the $P E$ Sox1 $(+35)$ modules} engineered within the Gata6-TAD. a, Detailed view of the bisulfite sequencing data presented in Fig. 3a, in which mESC (Day0) and AntNPC (Day5) differentiated from cell lines with the PE Soxl(+35)TFBS (left panel) or PE Soxl(+35)TFBS+CGI modules (right panel) inserted in the Gata6-TAD were analyzed. DNA methylation levels were measured using a forward bisulfite primer upstream of the insertion site and a reverse primer inside the TFBS module (Methods). The circles in the plots correspond to individual $\mathrm{CpG}$ dinucleotides located within the TFBS module. Unmethylated CpGs are shown in white, methylated CpGs in black and not-covered CpGs are shown in gray. 10 alleles (rows) were analyzed for each differentiation stage and cell line. b, Chromatin accessibility at the endogenous $P E$ Sox1(+35), the Gata6 TAD insertion site (primer pairs P1 and P2) and the Gata6 promoter were measured by FAIRE-qPCR in mESCs (left panels) and AntNPC (right panels) that were either WT (gray) or homozygous for the insertions of the different PE Sox1(+35) modules (i.e. TFBS (blue), CGI (yellow), TFBS+CGI (red)). FAIRE-qPCR signals were normalized against two negative control regions (Supplementary Data 1). Error bars correspond to standard deviations from technical triplicates. The location of the primer pairs P1 and P2 around the Gata6-TAD insertion site is represented as red arrows in the diagram shown to the right. c, DNA methylation and nucleosome occupancy at the TFBS module were simultaneously analyzed by NOME-PCR in ESC lines with the PE Soxl(+35)TFBS (left panel) or PE Soxl(+35)TFBS+CGI modules (right panel) inserted in the Gata6-TAD. In the upper panels, the black and white circles represent methylated or unmethylated CpG sites, respectively. In the lower panels, the blue or white circles represent accessible or inaccessible $\mathrm{GpC}$ sites for the $\mathrm{GpC}$ methyltransferase, respectively. Red bars represent regions large enough to accommodate a nucleosome and that are considered as inaccessible. The dotted line represents the region where the TFBS sequence starts. The primers used to amplify the TFBS sequences are shown as red arrows in the schematic diagrams, with one of the primers being located within the inserted TFBS and the other one immediately outside. The grey shaded area represent a nucleosome depleted region. d, Scatter plots showing population-averaged nucleosome occupancy (red) and DNA methylation (black) levels within the TFBS sequence in cells with either the PE Soxl(+35)TFBS (left panel) or PE Soxl(+35)TFBS+CGI (right panel) modules inserted within the Gata6-TAD. The grey shaded area represent a nucleosome depleted region. 
bioRxiv preprint doi: $\mathrm{https}: / /$ doi.org/10.1101/2020.08. 237768 ; this version posted August 5, 2020. The copyright holder for this preprint

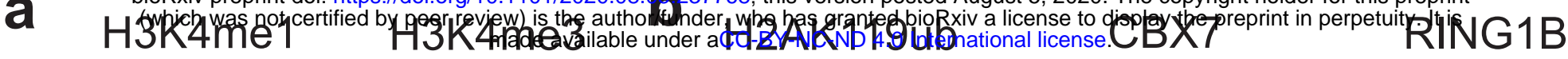
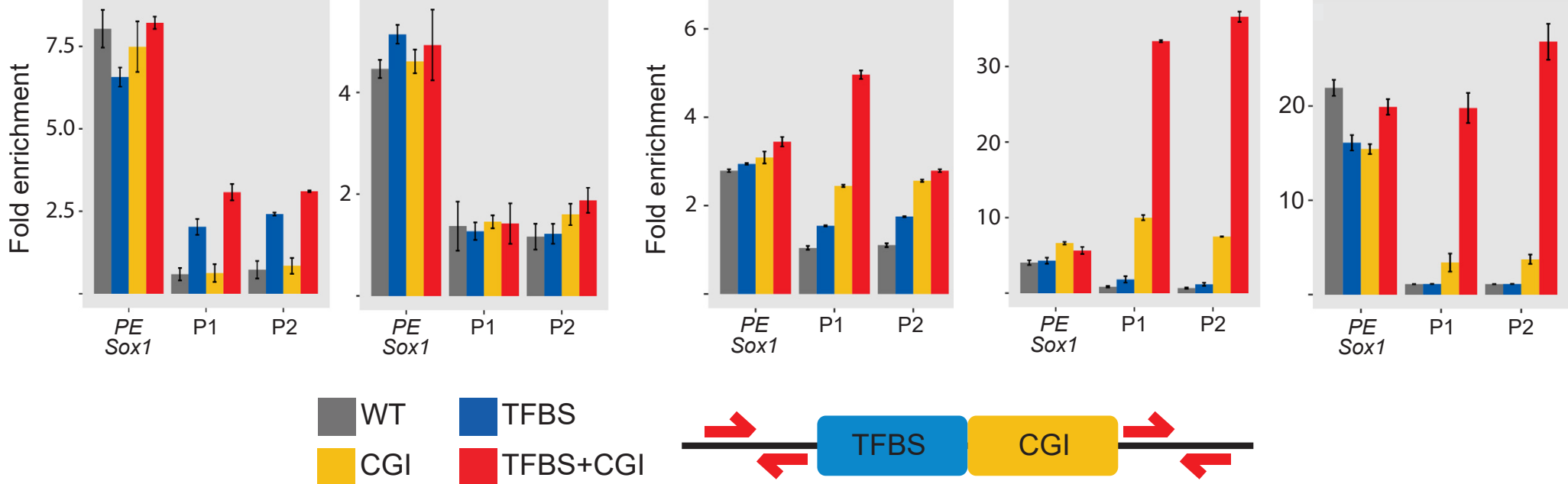

C

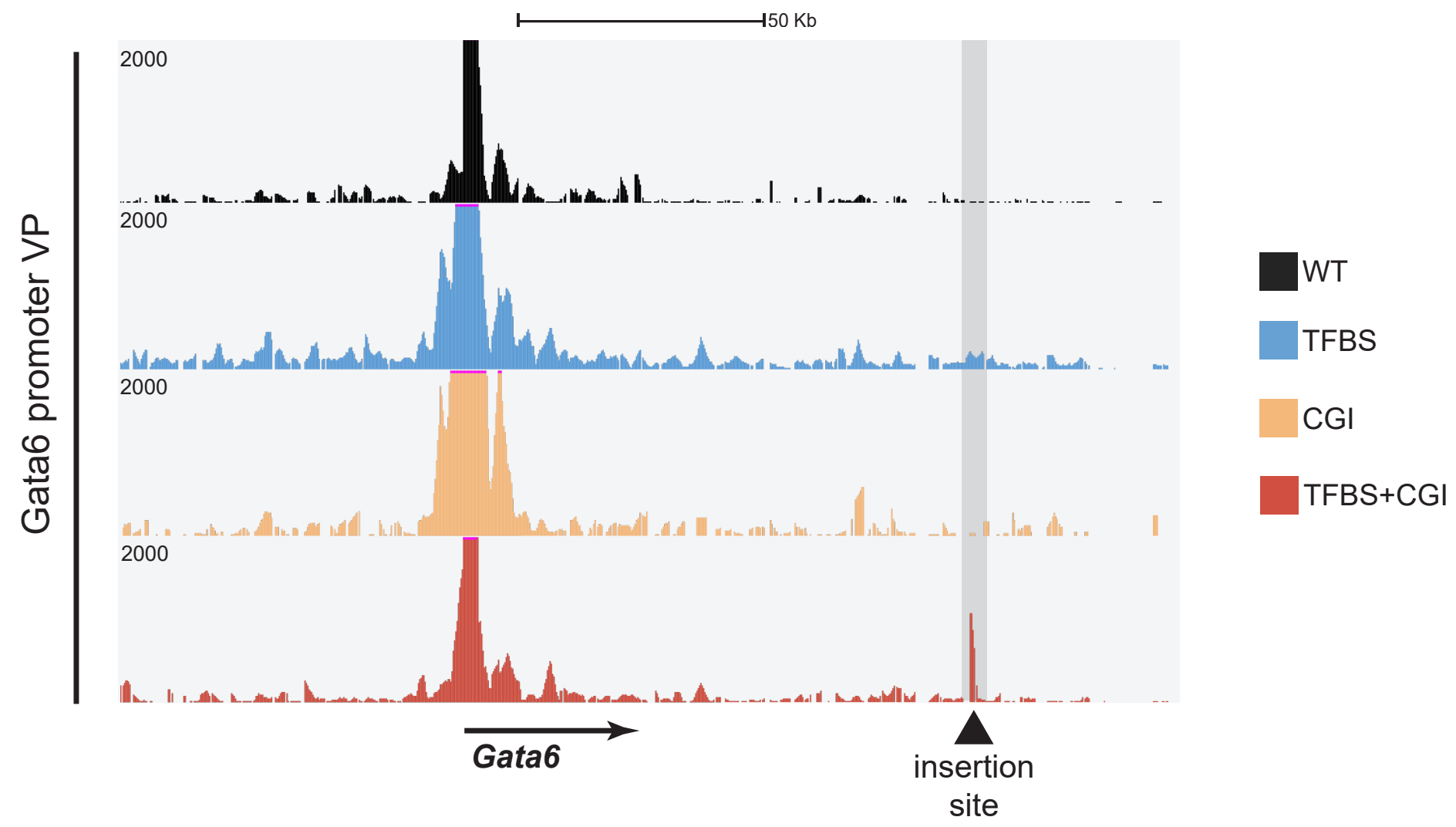

site 
Extended Data Fig.8| Chromatin and topological features of the PE Sox1(+35) modules engineered within the Gata6-TAD. a-b, H3K4me1 (a), H3K4me3 (a), H2AK119ub (b), CBX7 (b) and RING1B (b) levels at the endogenous PE Soxl(+35) and the Gata6-TAD insertion site (primer pairs P1 and P2) were measured by ChIP-qPCR in mESCs that were either WT (gray) or homozygous for the insertions of the different PE Sox1(+35) modules (i.e. TFBS (blue), CGI (yellow), TFBS+CGI (red)). ChIP-qPCR signals were normalized against two negative control regions (Supplementary Data 1). Error bars correspond to standard deviations from technical triplicates. The primers P1 and P2 around the Gata6-TAD insertion site are described in Fig 3b. c, 4C-seq experiments were performed using the Gata6 promoter as a viewpoint in AntNPC that were either WT (black) or homozygous for the insertions of the different PE Soxl(+35) modules (i.e. TFBS (blue), CGI (yellow), TFBS+CGI (red)). 


\section{Extended Data Fig.9| Generation and characterization of cell lines with engineered $P E$}

Sox1(+35) modules within the Grial-TAD. a, For the identification of $\mathrm{mESC}$ clonal lines with the desired insertion of the different PE Soxl(+35) modules, primer pairs flanking the insertion borders $(1+3$ and $4+2 ; 1+5$ and $6+2$; or $1+3$ and $6+2)$, amplifying potential duplications $(4+3,3+2$ and $4+1$; or $6+5,5+2$ and $6+1)$ and amplifying a large or small fragment depending on the absence or presence of the insertion $(1+2)$, respectively, were used. The PCR results obtained for WT mESC or two mESC clonal lines with homozygous insertions of the different $P E \operatorname{Soxl}(+35)$ modules (i.e. (i) $P E \operatorname{Soxl}(+35) T F B S$; (ii) $P E$ Soxl(+35)CGI; (iii) PE Soxl(+35)TFBS+CGI) in the Grial-TAD are shown. b, Independent biological replicate for the data presented in Fig. 4b. The expression of Grial and Soxl was measured by RT-qPCR in mESCs (left panels) and AntNPC (right panels) that were either WT (grey) or homozygous for the insertions of the different PE Soxl(+35) modules (TFBS (blue), CGI (yellow), TFBS+CGI (red)). For the cells with the PE module insertions, two different clonal lines (circles and diamonds) were studied in each case. For each cell line, two technical replicates of the AntNPC differentiation were performed. The plotted expression values for each clone correspond to the average and standard deviation (error bars) from three RT-qPCR technical replicates. Expression values were normalized to two housekeeping genes (Eefla and Hprt). c, Bisulfite sequencing analyses of ESC lines with the PE Soxl(+35)TFBS or PE Soxl(+35)TFBS+CGI modules inserted in the Grial-TAD. DNA methylation levels were measured using a forward bisulfite primer upstream of the insertion site and a reverse primer inside the TFBS module (see Methods). The circles shown in the left plots correspond to individual $\mathrm{CpG}$ dinucleotides located within the TFBS module: unmethylated CpGs are shown in white, methylated $\mathrm{CpGs}$ in black and not-covered $\mathrm{CpGs}$ are shown in gray. 10 alleles (rows) were analyzed for each differentiation stage and cell line. The plot on the right summarizes the DNA methylation levels measured within the TFBS in the mESC lines containing the PE Soxl(+35)TFBS and PE Soxl(+35)TFBS+CGI mESC inserts within the Grial-TAD. d-e, RNAP2 (d), MED1 (d), H3K27me3 (e) and H2AK119ub (e) levels at the endogenous PE Soxl(+35), the Grial-TAD insertion site (primer pairs P1 and P2) and the Grial promoter were measured by ChIP-qPCR in mESCs (left panels) and AntNPC (right panels) that were either WT (gray) or homozygous for the insertions of the different $P E$ Soxl(+35) modules (i.e. TFBS (blue), CGI (yellow), TFBS+CGI (red)). ChIP-qPCR signals were normalized against two negative control regions (Supplementary Data 1). Error bars correspond to standard deviations from technical triplicates. The location of the primers P1 
bioRxiv preprint doi: https://doi.org/10.1101/2020.08.05.237768; this version posted August 5, 2020. The copyright holder for this preprint (which was not certified by peer review) is the author/funder, who has granted bioRxiv a license to display the preprint in perpetuity. It is made available under aCC-BY-NC-ND 4.0 International license.

and $\mathrm{P} 2$ around the Grial-TAD insertion site is represented as red arrows in the diagram shown to the right. 
bioRxiv preprint doi: https://doi.org/10.1101/2020.08.05.237768; this version posted August 5, 2020. The copyright holder for this preprint

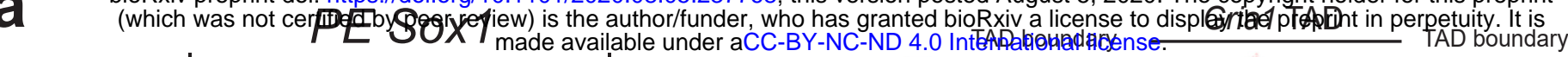

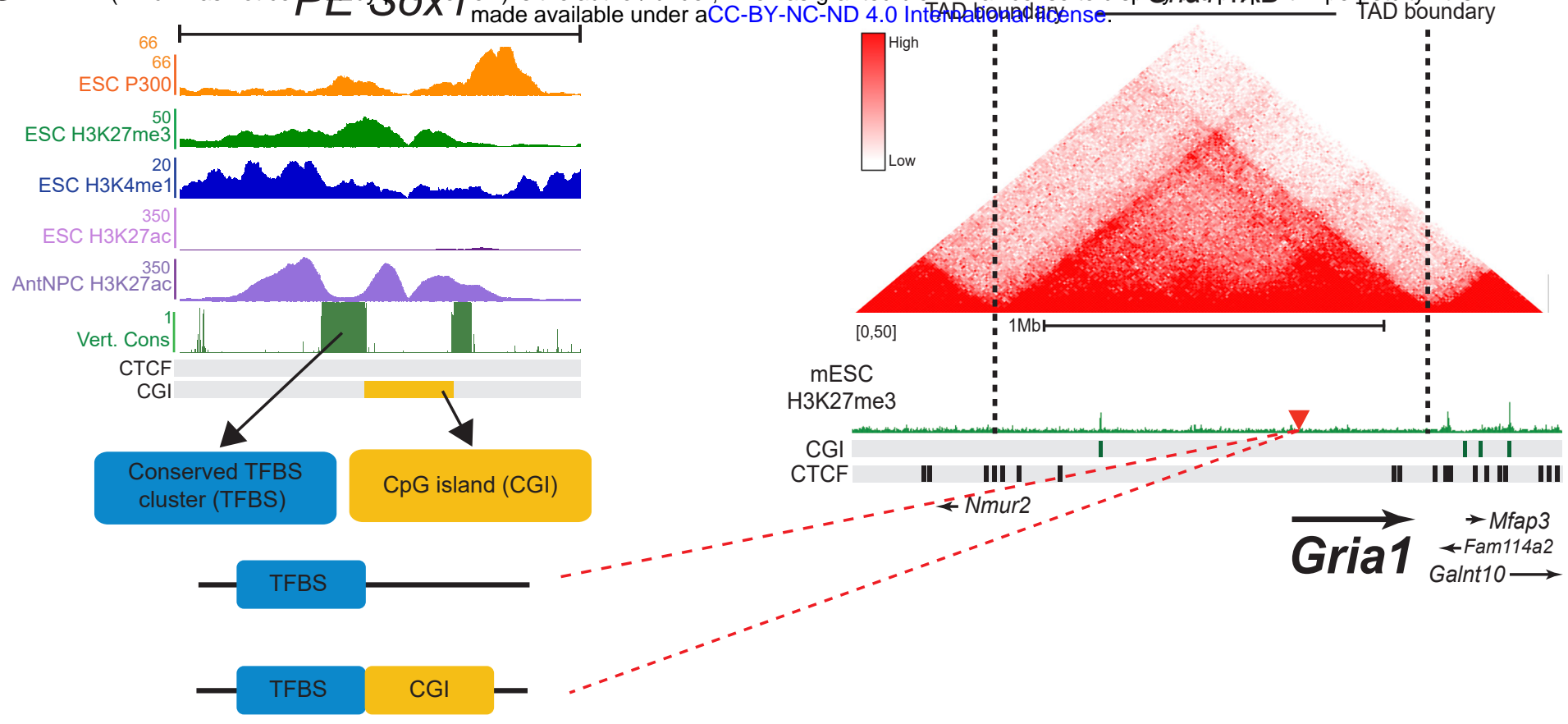

b

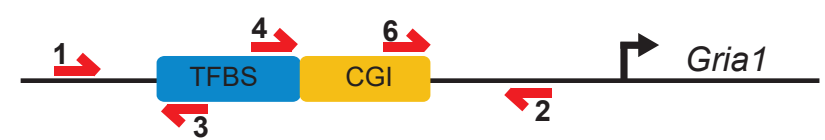

$1+2=b p($ TFBS $), 2148 \mathrm{bp}($ TFBS $+\mathrm{CGI})$ or $718 \mathrm{bp}(\mathrm{WT})$

$1+3=485 b p$

4+2 $=519 \mathrm{bp}($ TFBS $)$ or 14126bp $($ TFBS $+\mathrm{CGI})$

6+2 $=504 b p$

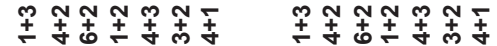

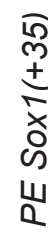

1

这
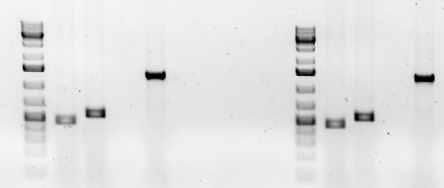

C

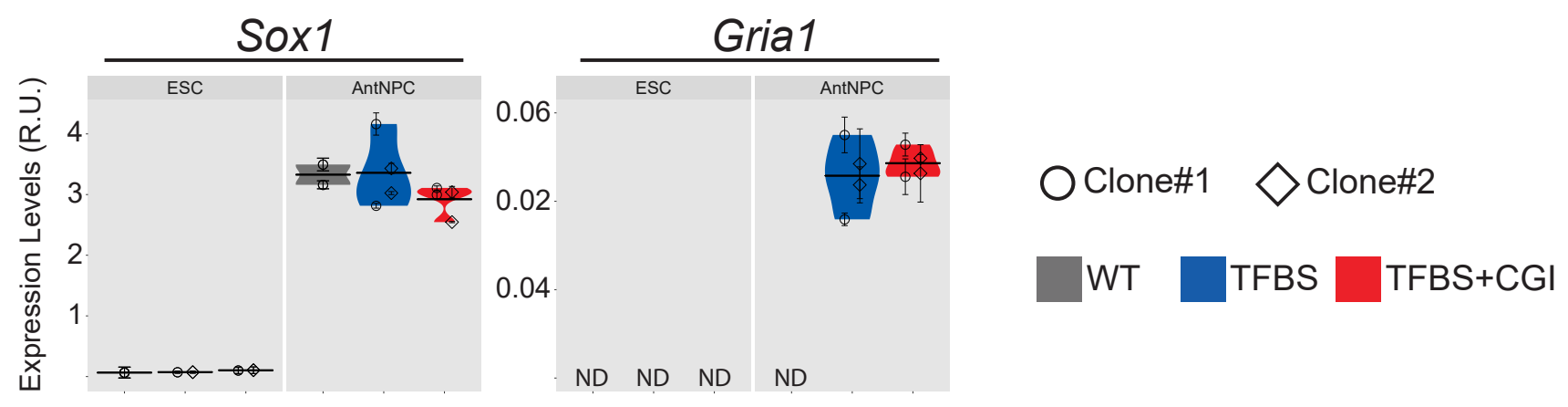

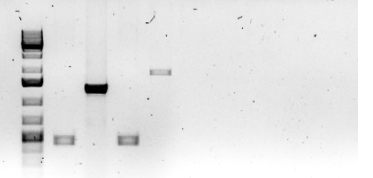

ox1

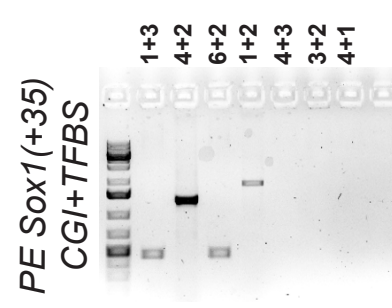

这 $\lessgtr_{500}^{1500}$

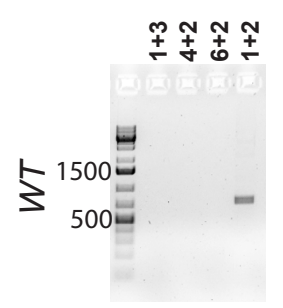

Clone\#1 $\diamond$ Clone\#2

WT

TFBS TFBS+CGI 


\section{Extended Data Fig.10| Modular engineering of the PE Sox1(+35) immediately upstream} of the Grial-TSS. a, Strategy used to insert the $P E$ Soxl $(+35) T F B S$ and $P E$ Soxl(+35)TFBS+CGI modules 380 bp upstream of the Grial TSS. The upper left panel shows a close-up view of the epigenomic and genetic features of the PE Soxl(+35). The represented CGIs correspond to those computationally defined in the UCSC browser according to the following criteria: GC content > 50\%; Length > $200 \mathrm{bp}$; $\mathrm{CpG}$ Observed to expected ratio $>0.6$. The lower left panel shows the two combinations of PE Soxl $(+35)$ modules (i.e. PE Soxl(+35)TFBS and PE Soxl(+35)TFBS\&CGI) inserted 380 bp upstream of the Grial TSS. The right panel shows the TAD in which Grial is included (i.e. Grial-TAD) according to publically available $\mathrm{Hi}-\mathrm{C}$ data ${ }^{33,65}$; TAD boundaries are denoted with dotted lines; H3K27me3 ChIP-seq signals in mESC are shown in green ${ }^{9}$; CGIs are indicated as green rectangles; CTCF binding sites $^{35}$ are indicated as black rectangles; the yellow triangle indicates the integration site of the PE Soxl(+35) modules, 380 bp upstream of the Grial TSS. b, For the identification of mESC clonal lines with the desired insertion of the different PE Sox $1(+35)$ modules, primer pairs flanking the insertion borders $(1+3$ and $4+2$; or $1+3$ and $6+2)$, amplifying potential duplications $(4+3,3+2$ and 4+1) and amplifying a large or small fragment depending on the absence or presence of the insertion $(1+2)$, respectively, were used. The PCR results obtained for WT mESC or two mESC clonal lines with homozygous insertions of the different $P E \operatorname{Soxl}(+35)$ modules (i.e. (i) $P E \operatorname{Soxl}(+35) T F B S$; (ii) $P E$ Soxl(+35)TFBS+CGI) 380 bp upstream of the Grial TSS are shown. c, Independent biological replicate for the data presented in Fig. 4f. The expression of Grial and Soxl was measured by RT-qPCR in mESCs (left panels) and AntNPC (right panels) that were either WT (grey) or homozygous for the insertions of the different PE Soxl $(+35)$ modules 380 bp upstream of the Grial TSS (TFBS (blue); TFBS+CGI (red)). For the cells with the PE module insertions, two different clonal lines (circles and diamonds) were studied in each case. For each cell line, two technical replicates of the AntNPC differentiation were performed. The plotted expression values for each clone correspond to the average and standard deviation (error bars) from three RT-qPCR technical replicates. Expression values were normalized to two housekeeping genes (Eefla and Hprt). 
bioRxiv preprint doi: https//doi.org/10.1101/2020 08 05.237768; this version posted August 5, 2020. The copyright holder for this preprint (which was not certified by peer review) is the author/funder, who has granted bioRxiv a license to display the preprint in perpetuity. It is H3K27ac
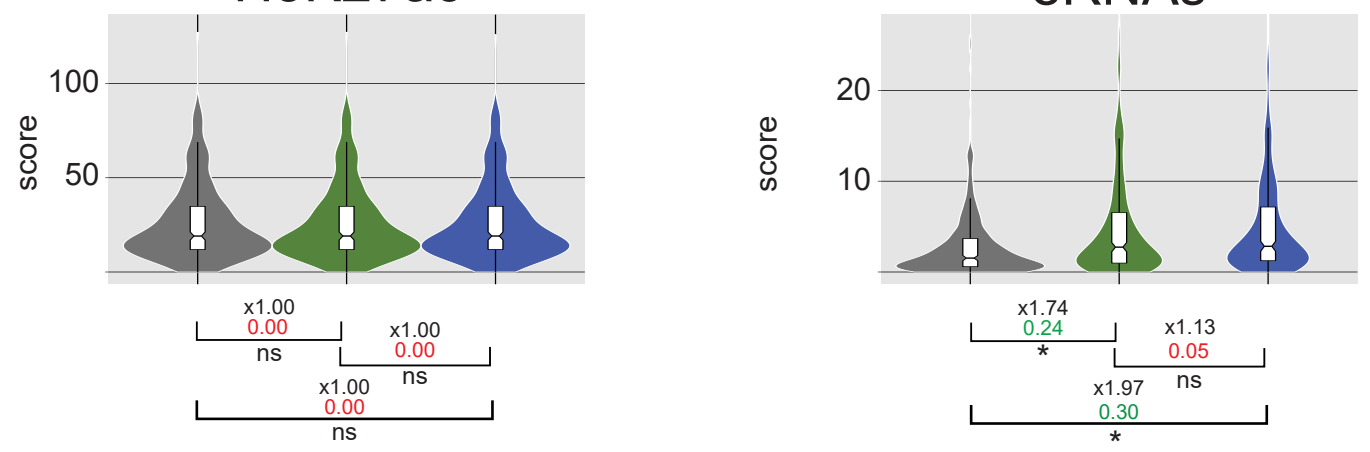

Class I

Class II

Class III
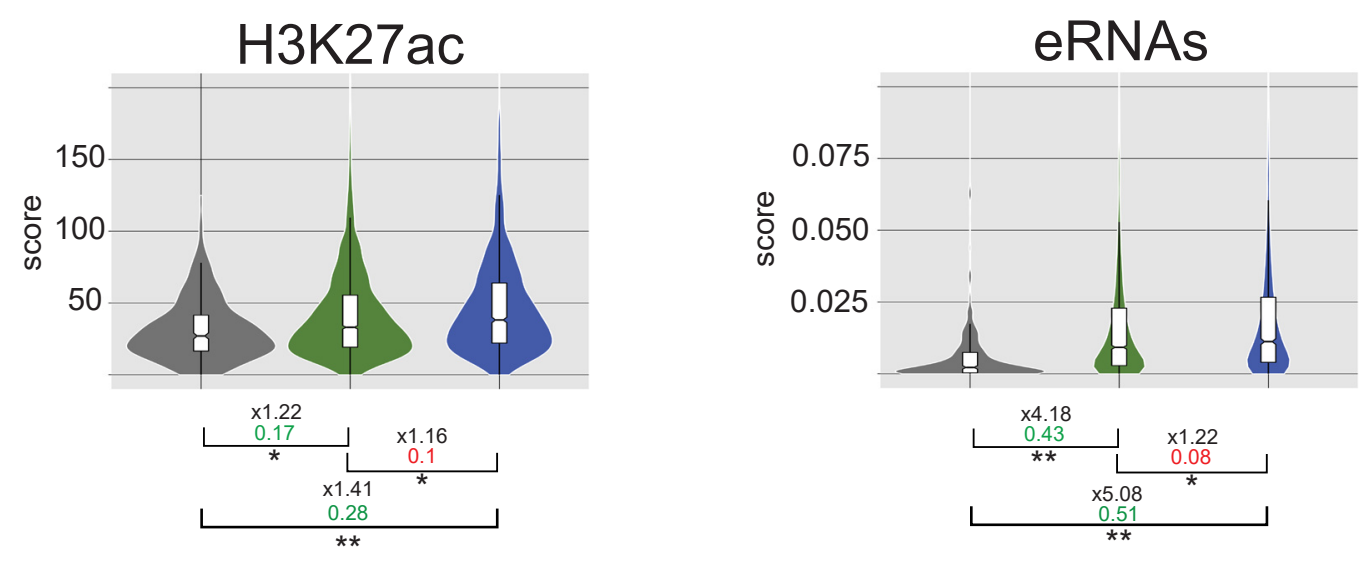

Class I

Class II

Class III
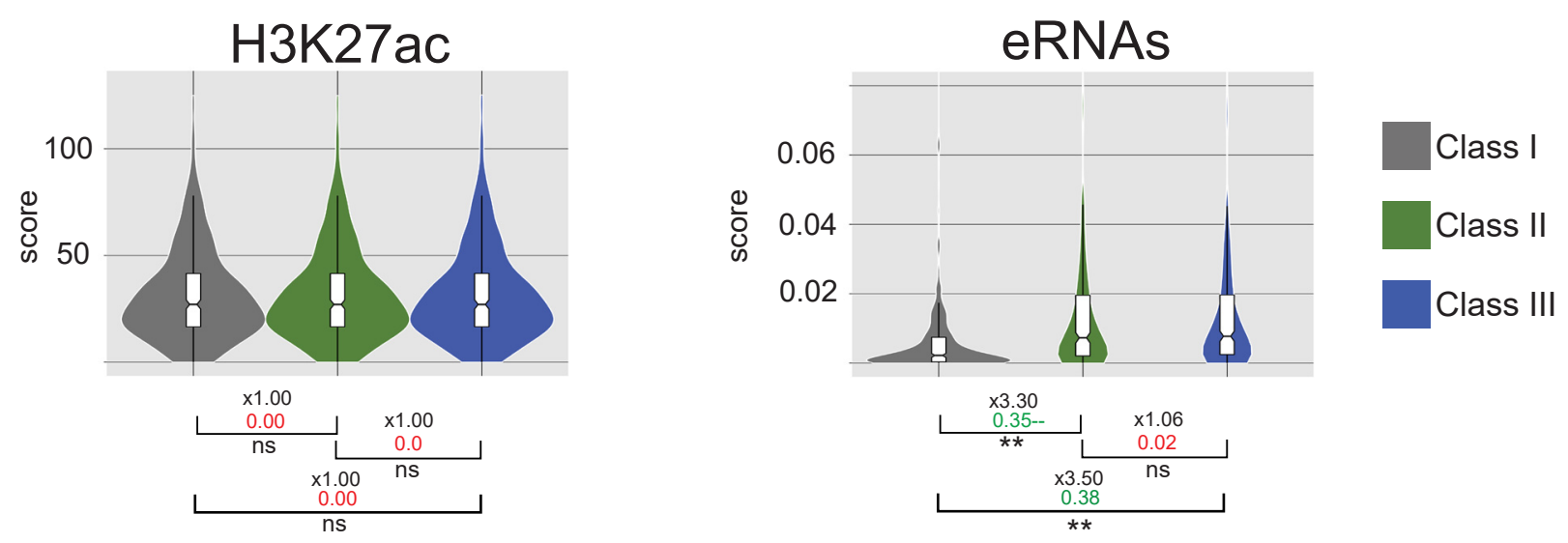
Extended Data Fig.11| H3K27ac and eRNA levels for different classes of active enhancers in mESC. a-c, Active enhancers identified in $\mathrm{mESC}$ based on the presence of distal H3K27ac peaks (see Methods) were classified into three categories: Class I correspond to active enhancers located in TADs containing only poorly expressed genes (all genes with $<0.5$ FPKM); Class II correspond to active enhancers located in a TAD with at least one gene with expression levels >10 FPKM; Class III correspond to active enhancers whose closest gene in their same TAD has expression levels >10 FPKM). The violin plots show the H3K27ac (left) and eRNA (right) levels for each of these active enhancer categories in mESC. On the bottom of each plot, the asterisks indicate P-values calculated using unpaired Wilcoxon tests with Bonferroni correction for multiple testing $\left(* *=\right.$ p.val $<1 \mathrm{e}^{-10}$; $*$ p.val $<$ 0.05); the numbers in black indicate the median fold-changes between the indicated groups; the coloured numbers correspond to Cliff Delta effect sizes: negligible (red) and nonnegligible (green). In (a), the H3K27ac ChIP-seq data was obtained from ${ }^{9}$ and the PRO-seq data to measured eRNA levels was obtained from ${ }^{66}$. In (b-c), the H3K27ac ChIP-seq data was obtained from ${ }^{69}$ and the PRO-seq data to measured eRNA levels was obtained from ${ }^{109}$. In (a) and (c), eRNA levels for the three enhancers classes are compared after correcting for H3K27ac differences (see Methods). 
a (which was not certified by peer review) is the author/funder, who has granted bioRxiv a license to display the preprint in perpetuity. It is

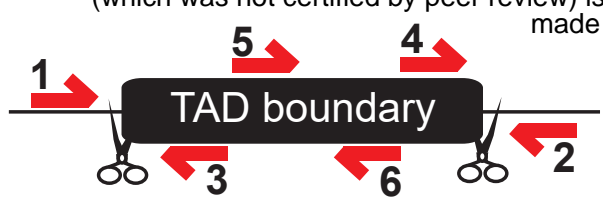

$1+2=37465 \mathrm{bp}(\mathrm{WT})$ or $556 \mathrm{bp}(\mathrm{de} / 36)$

$1+3=484 \mathrm{bp} \quad 2+4=528 \mathrm{bp}$

$5+6=698 \mathrm{bp}$
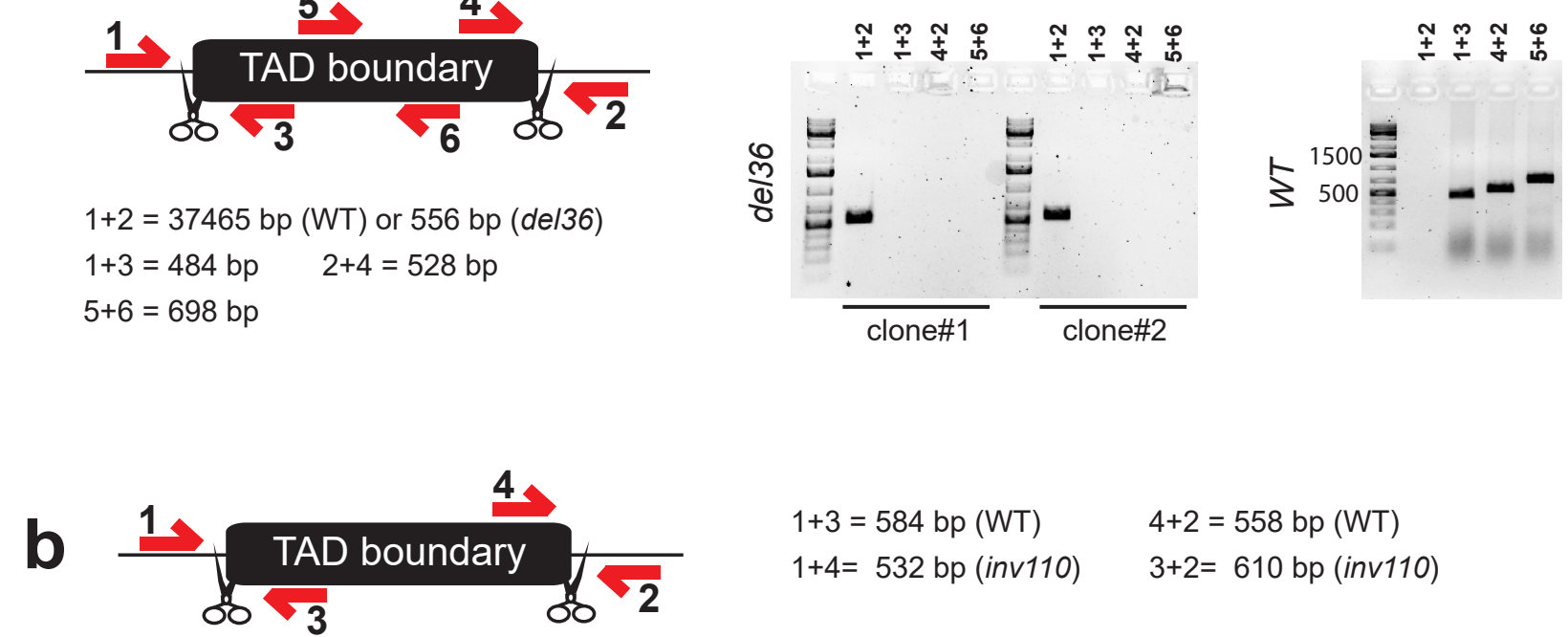

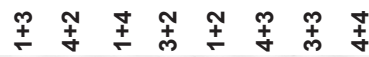

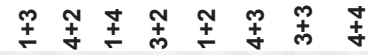

$1+3=584 \mathrm{bp}(\mathrm{WT})$

$4+2=558 \mathrm{bp}(\mathrm{WT})$

$1+4=532 \mathrm{bp}($ inv110)

$3+2=610 \mathrm{bp}($ inv110 $)$

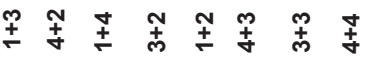

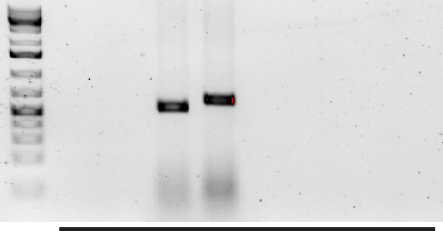

clone\#1

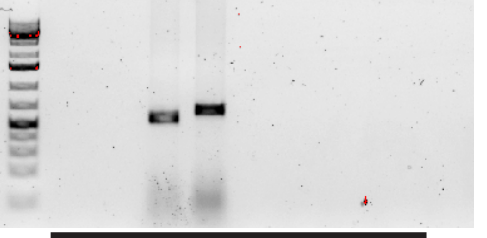

clone\#2

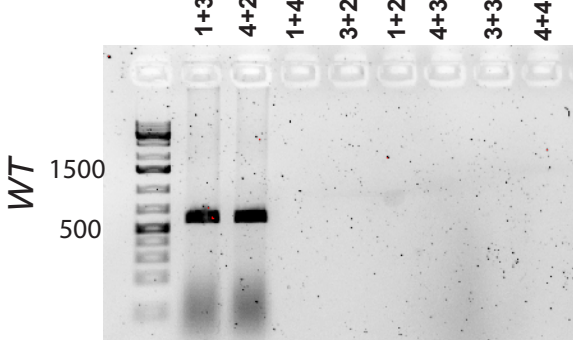

TAD

C

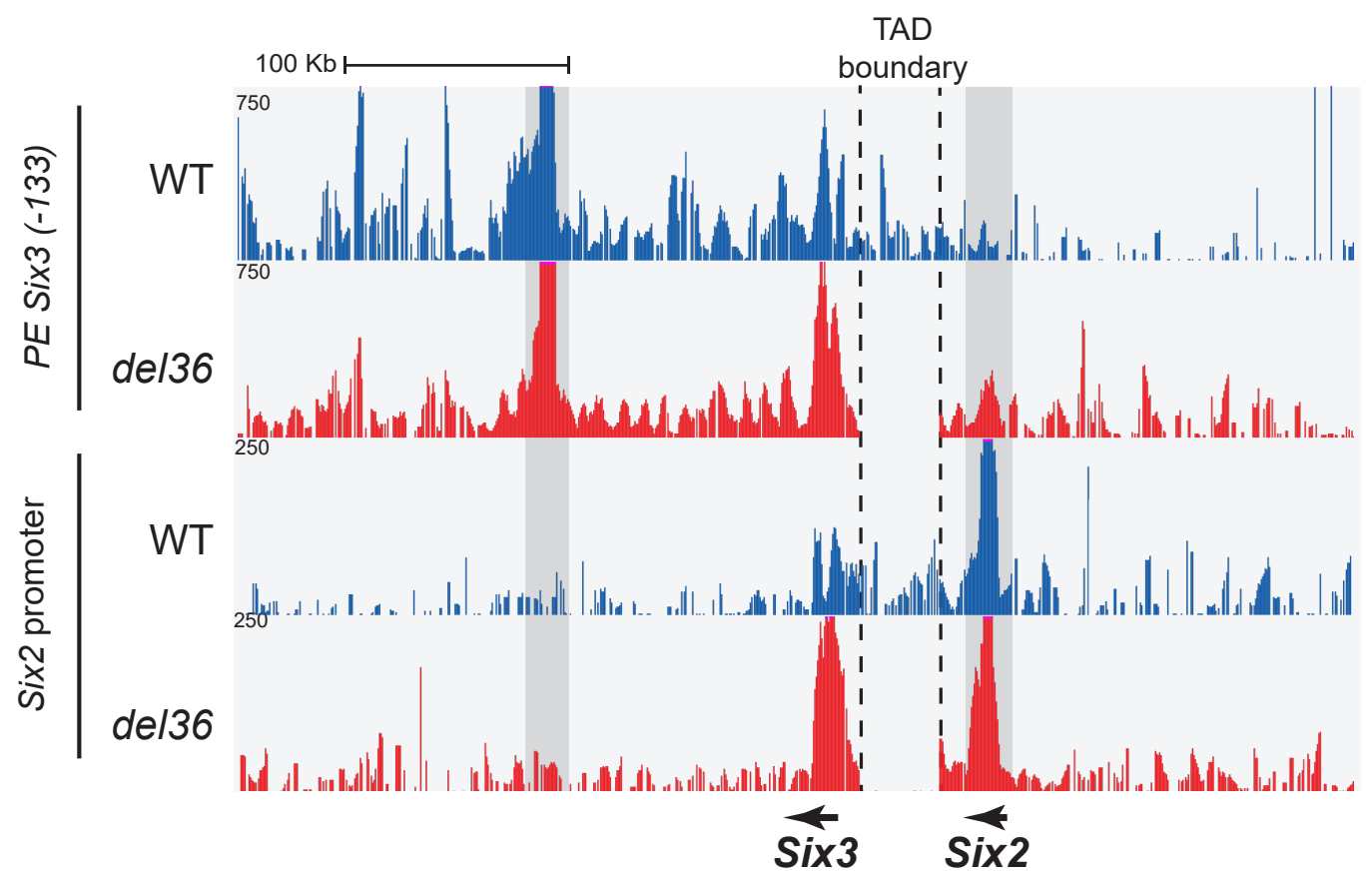


Extended Data Fig.12| Generation of mESC lines with structural variants within the

Six3/Six2 locus. a, For the identification of mESC lines with the Six3/Six2 TAD boundary deletion, primer pairs flanking the deleted region $(1+3$ and $4+2)$, amplifying the deleted fragment (5+6) and amplifying a large or small fragment depending on the absence or presence of the deletion $(1+2)$, respectively, were used. The PCR results obtained for two mESC clonal lines with $36 \mathrm{~Kb}$ homozygous deletions (del36) are shown. b, For the identification of mESC lines with the Six3/Six2 inversion, primer pairs flanking the inverted region $(1+3,4+2,1+4$ and $3+2)$ and amplifying potential duplications $(4+3,3+3$ and $4+4)$ were used. The PCR results obtained for two mESC clonal lines with $110 \mathrm{~Kb}$ homozygous inversions (inv110) are shown. c, 4C-seq experiments were performed using the PE Six3(133) (upper panels) or the Six2 promoter (lower panels) as viewpoints in mESCs that were either WT (blue) or homozygous for the del36 deletion (red). 